\title{
Therapy-induced senescence in normal tissue promotes breast cancer metastasis
}

${ }^{1}$ Douglas W. Perkins, ${ }^{1}$ Syed Haider, ${ }^{1}$ David Robertson, ${ }^{1}$ Richard Buus, ${ }^{1}$ Lynda O'Leary and

\author{
1,* Clare M. Isacke
}

\author{
${ }^{1}$ The Breast Cancer Now Toby Robins Research Centre, Institute of Cancer Research, \\ London
}

*Corresponding author: Clare M. Isacke, The Breast Cancer Now Toby Robins Research Centre, Institute of Cancer Research, 237 Fulham Road, London SW3 6JB, UK.

Tel: +44 207153 5510; Fax: 0207153 5340; Email: clare.isacke@icr.ac.uk 


\section{Summary}

Disseminated tumour cells, particularly in ER+ breast cancers, typically exhibit a period of dormancy that renders them insensitive to targeting by chemotherapy. Additionally, chemotherapy treatment can result in normal tissue damage, including the induction of cellular senescence. Using mouse and human breast cancer models, we demonstrate that systemic chemotherapy administration results in accumulation of long-lived senescent stromal fibroblasts and promotes metastatic outgrowth. Chemotherapy-induced senescent fibroblasts upregulate a senescence associated secretory phenotype (SASP) that accelerates 3D tumour spheroid growth by stimulating mitogenic signalling. Senolytic drugs can effectively eliminate chemotherapy-induced senescent fibroblasts in vitro, but show only modest efficacy in vivo, at least in part due to the upregulation of resistance mechanisms. In conclusion, systemic chemotherapy can establish a productive microenvironment for colonisation and outgrowth of disseminated cancer cells, however, optimisation of senotherapies for effective targeting of senescent fibroblasts is required to establish them as useful additions to standard chemotherapy.

\section{Introduction}

Over the past decade there has been extensive research into understanding the functional consequences of cellular senescence in vivo. There is now compelling evidence that senescent cells, which accumulate as a result of natural ageing (Baker et al., 2016; Baker et al., 2008), underpin a range of age-related pathological conditions including idiopathic pulmonary fibrosis, atherosclerosis, osteoarthritis and renal failure (Baker et al., 2011; Childs et al., 2016; Schafer et al., 2017). Despite being long-lived and non-proliferative, senescent cells are far from static and inert. Rather, they are highly metabolically active and have elevated expression of the senescence-associated secretory phenotype (SASP), a diverse cocktail of factors responsible for many of their non-cell-autonomous functions. As a result, there is increasing interest in strategies designed to selectively kill senescent cells, with the 
aim of alleviating chronic and ageing-related disorders, whilst leaving normal, healthy cells unaffected (Cai et al., 2020; Childs et al., 2017; Xu et al., 2018).

In the context of cancer, the picture is more complex. Tumour cells can enter a state of senescence as a consequence of oncogene activation or therapeutic insult. Initially, tumour cell senescence was considered a beneficial therapeutic outcome (Dörr et al., 2013; Serrano et al., 1997). However, it now accepted that senescent tumour cells can provide a reservoir for potential cancer relapse should they manage to subsequently reinitiate proliferation (Milanovic et al., 2018). Further, multiple studies have revealed that increased normal tissue cell senescence can promote tumour progression (Alspach et al., 2014; Gonzalez-Meljem et al., 2018), creating a link between ageing and cancer (Krtolica et al., 2001). In addition to age-related senescence, normal tissue senescence is elevated following systemic chemotherapy treatment, even in young animals (Demaria et al., 2017). These latter findings are corroborated by clinical studies of patients receiving chemotherapy or radiotherapy, who were shown to have increased markers of molecular age, especially in haematopoietic tissues (Sanoff et al., 2014; Scuric et al., 2017). Further, survivors of childhood cancers who received chemotherapy as part of their treatment, have an increased risk of developing late onset pathologies as adults, including increased incidence of secondary cancers (Guida et al., 2020; Oeffinger et al., 2006).

For breast cancer patients, prevention of local recurrence is achieved by surgery and localised radiotherapy, whilst systemic therapies are aimed, almost exclusively, at limiting the outgrowth of disseminated disease. In the metastatic setting the ratio of tumour cells compared to surrounding secondary site tissue is very low; hence the stromal milieu has a profound impact on the fate of disseminated tumour cells and disease outcome. Here we have investigated the effect of systemic chemotherapy on the induction of normal tissue senescence and the role of senescent stromal cells in shaping disease progression. 


\section{Results}

\section{Prior chemotherapy promotes metastatic tumour growth}

To test the hypothesis that systemic chemotherapy treatment can prime the host tissue microenvironment, making it a more receptive niche for metastatic tumour development, we employed experimental metastasis assays that discriminate between the effects of the chemotherapy treatment on the stroma from any direct effects on the tumour cells (Figure 1a). Naive BALB/c mice were treated with a course of chemotherapy; consisting of four doses over two weeks of doxorubicin combined with cyclophosphamide. This schedule reflects the cyclic delivery of chemotherapy in breast cancer patients in the clinic and has been demonstrated previously to have anti-tumour efficacy in syngeneic mouse models (Ashenden et al., 2017). Mice were inoculated intravenously with syngeneic tumour cells 7 days after the last treatment (Figure $1 \mathrm{~b}-\mathrm{d}$ ). The 7 -day recovery period was chosen to allow for clearance of the chemotherapeutic agents (Johansen, 1981; Park et al., 2012) and to allow for the repopulation of circulating lymphocytes (Saida et al., 2015) (see next section).

Breast cancer dormancy, where metastatic disease remains undetected for many years after treatment of the primary tumour, represents a major clinical challenge (Dittmer, 2017). Therefore, we selected the D2.OR cell line, because it exhibits a dormant-like phenotype in vivo (Barkan et al., 2008; Morris et al., 1994). 7 days after D2.OR tumour cell inoculation, single tumour cells are found scattered throughout the lung tissue of vehicletreated mice, whereas discrete tumour lesions are readily detectable in the mice receiving prior chemotherapy (Figure 1b). By 98 days after tumour cell inoculation, this translates into a significantly increased lung tumour burden in chemotherapy-treated mice (Figure 1c). To validate these findings, this experiment was repeated using the related but more aggressive D2A1 cell line (Morris et al., 1994) which, although showing limited ability to spontaneously metastasise, readily forms metastatic lesions when injected intravenously (Jungwirth et al., 2018). Metastatic lesions are detectable in the vehicle-alone group 15 days after intravenous inoculation, however, the size of tumour nodules is significantly increased in the mice which received prior chemotherapy treatment (Figure S1). 
Despite having a 7 day intermission between the end of chemotherapy treatment and inoculation of tumour cells, and the finding that lung tissue from non-tumour bearing mice sacrificed 1, 3, 7 or 28 days after the final vehicle or chemotherapy treatment show no significant change in the number of CD4+ and CD8+ T cells (Figure S2a); it remained a concern that chemotherapy could promote metastatic colonisation through an effect on the immune system. Therefore, the experimental approach outlined in Figure 1a was repeated using immunocompromised NOD RAG gamma (NRG) mice inoculated with human oestrogen receptor-positive $(E R+) Z R-75-1$ breast cancer cells. This cell line was chosen as it has also been reported to exhibit a dormant phenotype in in vivo models (Gawrzak et al., 2018). Again there is a striking enhancement of metastatic colonisation in the mice that had received prior chemotherapy. Unexpectedly, whilst intravenously injected cells initially disseminated to the lungs (Figure S2b), the majority of subsequent metastatic burden manifests in the liver (Figure 2d). Lung and bone metastasis was also significantly increased, albeit at reduced abundance compared to the liver (Figure S2c). These effects were recapitulated in ex vivo assays where we observed a significant increase in the number of viable ZR-75-1 cells surviving 10 days after seeding onto ex vivo organotypic lung slices derived from chemotherapy-treated compared to vehicle-treated mice (Figure S3).

\section{Chemotherapy treatment causes long-term normal tissue damage}

To examine in more detail the normal tissue changes elicited by chemotherapy treatment, non-tumour bearing, immune competent mice were treated with a course of chemotherapy between Day 1 and 14, then culled at five timepoints, between Day 15 and 105 (Figure 2a). Staining of lung tissue for phospho-yH2Ax $\left(\mathrm{P}_{\mathrm{H}} \mathrm{H} 2 \mathrm{Ax}\right)$, a marker of DNA damage response, revealed a sharp increase in $\mathrm{PyH} 2 \mathrm{Ax}$-positive cells in the lungs on Day 15, which peaks at Day 17 and then decreases to background levels by Day 42 (Figure 2b). Persistent DNA damage response signalling can induce permanent cell cycle arrest and senescence (Fumagalli et al., 2014; Rodier et al., 2009). Staining of lung sections for p21WAF1 (p21), a marker of DNA damage-induced senescence (de Carné Trécesson et al., 2011; Jurk et al., 
2012; Passos et al., 2010), revealed that induction of p21 expression is delayed with no increase above background until Day 17. p21 levels then remain elevated until Day 42, even when no PyH2Ax-positive cells are detected (Figure 2b). Equivalent results were obtained in an independent experiment where mice were sacrificed 21 days following chemotherapy treatment (Day 35) (Figure 2c). Moreover, RTqPCR analysis reveals a significantly increased expression of canonical SASP factors in the lung tissue of the chemotherapytreated mice (Figure 2d). Using freshly extracted RNA, a broader gene expression analysis was performed using the NanoString mouse PanCancer Immune and PanCancer Pathways panels (Figure 2e; Figure S4; Tables S1,S2). By principal component analysis (PCA) the vehicle-treated mice cluster separately from the chemotherapy-treated mice, implying global differences across the 750 target gene panels (Figure 2e; Figure S4). Importantly, a top hit within the significantly upregulated genes in chemotherapy-treated mice is Cdkn1a (p21), supporting the immunohistochemical staining and RTqPCR results (Figure $2 b-d$ ). Similarly, increased Mmp3, Cdkn2a, I/6 and Cxcl1 expression was detected, however, the adjusted $p$-values for $C d k n 2 a, I / 6$ and $C x c / 1$ are not significant. The significantly upregulated genes in the chemotherapy-treated samples cluster in pathways associated with pro-inflammation, apoptosis, cytokines and chemokines, and pro-cancer progression indicating that chemotherapy treatment creates a favourable, tumour-promoting microenvironment. These upregulated genes include: the II6 family cytokine Lif, which promotes cell proliferation via STAT3 signalling and has been reported to promote breast cancer metastasis (Li et al., 2014; Niwa et al., 2009); Cxcl10, a SASP factor reported to enhance breast cancer lung metastasis (Pein et al., 2020; Perrott et al., 2017); the pro-tumourigenic factor Wnt5a (Azazmeh et al., 2020),(Kobayashi et al., 2018); as well as Cc/2 (MCP1), a potent macrophage chemoattractant, reported to play a role in cancer progression (Acosta et al., 2013; Park et al., 2012; Sanoff et al., 2014), and the related factors Ccl7 (MCP3) and Ccl8 (MCP2). In contrast, downregulated genes clustered primarily in humoural immunity pathways such as the B-cell surface antigens Cd79b, Ms4a1, and Cd19 and the B-cell lineage specific Pax5. 


\section{Therapy-induced fibroblast SASP promotes tumour cell growth}

Amongst the increased levels of senescence and pro-tumourigenic factors in the gene expression analysis are genes associated with extracellular matrix remodelling and fibrosis (Figure 2e; Figure S4), including collagens type Va1 (Col5a1) and type IVa1 (Col4a1), Mmp3, Thbs1 (thrombospondin1), and Tnc (tenascin C) (Walraven and Hinz, 2018). Tissue fibrosis is associated with increased tumourigenicity in multiple cancer types (Barkan et al., 2010; Cox and Erler, 2014) and senescent fibroblasts are a primary cell-type responsible for this pathological phenotype (Schafer et al., 2017).

To elucidate whether senescent fibroblasts are present in our model of chemotherapy-induced tissue damage, lung sections from vehicle or chemotherapy-treated mice were co-stained for p21 alongside cell-type markers for myofibroblasts (alpha-smooth muscle actin, aSMA), epithelial cells (EpCAM) and endothelial cells (endomucin) (Figure 3a). Consistent with the data presented in Figure 2, significantly increased numbers of p21-positive cells are observed in lung tissue of the chemotherapy-treated mice (Figure 3a, middle panel). Since p21-positive cells in the lungs of vehicle-treated mice are scarce, very few double-positive cells of any type are identified. In contrast, in the chemotherapy-treated lung tissue, p21-positive cells are readily identified with the greatest increase being in double-positive p21 aSMA stained cells (Figure 3a, right panel). The identification of p21+ aSMA+ cells in chemotherapy-treated lung tissue was validated in immunohistochemical staining of lung sections from an independent experiment (Figure 2b). By immunofluorescence and immunohistochemistry, the majority of aSMA staining in vehicletreated lungs is localised to the vasculature, consistent with aSMA expression by pericytes and perivascular-localised fibroblasts. Whereas, in chemotherapy-treated lungs, p21 aSMA double positive cells are detected both associated with, and distant from, the vasculature, indicating that systemic chemotherapy drives the accumulation of senescent fibroblasts in the lung tissue (Figure 3a,b). 
Treatment of mouse or human fibroblasts in vitro for 24 hours with doxorubicin, followed by culture in the absence of doxorubicin induces a robust cell cycle arrest and positive staining using the senescence associated $\beta$-galactosidase assay (SA $\beta$-Gal) assay (Figure 3c,d; Figure S5a). In an independent approach, fibroblasts were transfected with a senescence reporter construct pmiR146a-GFP (Kang et al., 2015), in which GFP expression is driven by the senescence-associated microRNA146a promoter. Doxorubicin treatment results in increased expression of the senescence reporter, which is maintained for up to 20 days following drug withdrawal. Of note, in these assays doxorubicin-induced senescent human fibroblasts survive in culture for prolonged periods of time (> 2 months (Figure S5b) and $>6$ months (data not shown)). At longer time points, expression of the pmiR146a-GFP senescence reporter diminishes and cells accumulate lipofuscin granules, a marker of longterm senescence (Brunk and Terman, 2002; Georgakopoulou et al., 2013) (Figure S5b). These in vitro data, combined with the analysis of chemotherapy-treated lung tissue, indicate that senescent fibroblasts represent a long-lived product of chemotherapy treatment that could persist even when the tumour has been reduced or eliminated. To model this in more complex in vitro models, RFP-tagged D2A1 mouse mammary tumour cells were admixed with human miR146a-transfected MCR5 fibroblasts and co-seeded into ultra-low attachment U-bottom 96-well plates to form 3D co-culture spheroids. Treatment with increasing concentrations of doxorubicin results in effective elimination of the tumour cells, whilst the admixed fibroblasts are induced to express markers of senescence and persist until the end of the assay (Figure 4e).

Chemotherapy-induced senescent mouse fibroblasts show a robust upregulated expression of canonical SASP transcripts (Figure 4a), accompanied by increased secretion of SASP factors into the culture medium (Figure 4b). These findings were replicated in chemotherapy-induced senescent primary human lung fibroblasts (Figure 4c,d; Figure S5c). Incubation of 3D mouse or human tumour spheroids with conditioned medium harvested from doxorubicin-induced senescent mouse and human fibroblasts results in a striking increase in spheroid size compared to incubation with conditioned medium from control 
fibroblasts (Figure 4e; Figure S6a). To confirm that the increased growth of the spheroids results from increased tumour cell proliferation, as opposed to decreased cell death, two approaches were taken. First, tumour cell colony formation assays show no significant difference in colony number in the presence of senescent fibroblast conditioned medium, but there is a notable increase in colony size (Figure S6b). Second, tumour spheroids were fixed and embedded, and sections were stained for the proliferation marker Ki67 (Figure 4f). As the experiments were conducted in low (2\%) serum conditions, few if any Ki67-poistive cells are detected in spheroids cultured with control conditioned medium. By contrast, spheroids incubated with senescent fibroblast conditioned medium are larger in size and have a ring of Ki67-positive cells around their periphery. To investigate the mechanism underlying this increased 3D tumour spheroid proliferation, D2A1 mouse or ZR-75-1 human tumour spheroids were treated with conditioned medium for 30 minutes, lysed and then assayed by phosphoprotein array. Increased phosphorylation of several key mitogenic factors is observed in the spheroids incubated with senescent fibroblast conditioned medium. In particular there is increased phosphorylation of Tyr701 STAT1, Tyr705 STAT3 and pan-SRC in the D2A1 spheroids (Figure 4f) and increased phosphorylation of Ser473 AKT, Thr202/Tyr204 ERK p42/p44 and Tyr705 STAT3 in the ZR-75-1 spheroids (Figure S6c). These findings are consistent with the activation of STAT3 signalling downstream of SASP cytokines such as IL6 and LIF (Hodge et al., 2005; Li et al., 2014; Niwa et al., 2009), whose expression is upregulated in senescent fibroblasts, promoting proliferation of mouse and human tumour cells in both in vitro and in vivo models.

\section{Senescent fibroblasts can be selectively eliminated in vitro}

Prolonged survival of senescent cells requires the avoidance of apoptotic-mediated cell death commonly by upregulated expression of anti-apoptotic BCL2 family members (de Carné Trécesson et al., 2011; Malaquin et al., 2020). BCL2 family proteins bind to and inhibit $\mathrm{BH}$-initiator proteins such as BAD, which would otherwise activate the apoptotic effector protein BAX to trigger caspase activation and mitochondrial outer membrane 
permeabilisation (Willis et al., 2003). Consequently, agents which target the BCL2 family members, such as BCL2, BCLxL, BCLw and MCL1 (Figure 5a), have been assessed for their efficacy as senolytic agents. In particular, navitoclax, which targets BCL2, BCLxL and BCLw, has been shown to selectively kill senescent cells in vivo (Chang et al., 2016). However, there has been little focus on their use in targeting therapy-induced senescent fibroblasts. As the gene expression analysis reveals significantly increased Bax expression in the lung tissue from chemotherapy-treated mice (Figure 2e), we determined whether BCL2 family inhibitors could effectively eliminate senescent cells in our models.

Fibroblasts induced into senescence by 24 hour doxorubicin treatment and then incubated for 10 days in the absence of drug are effectively killed by subsequent treatment with either navitoclax or the BCLxL specific inhibitor A-1155463 (A115) whereas these inhibitors have limited effect on control vehicle-treated fibroblasts (Figure 5b). By contrast, treatment with either the BCL2-only inhibitor venetoclax or the MCL1 inhibitor UMI-77 has no impact on senescent or control fibroblast viability. Comparable results were obtained using human IMR90 fibroblasts, mouse 3T3 fibroblasts, and fibroblasts induced into senescence through docetaxel treatment (Figure S7a-c). A concern in these assays is that CellTiter-Glo monitors ATP levels in the cell and it is likely that proliferating and senescent cells have different basal ATP metabolism. Importantly, equivalent results are obtained using nonenzymatic crystal violet staining (Figure 5c; Figure S6a). As MCL1 exhibits some functional redundancy with other BLC2 family members (Eichhorn et al., 2014), the effect of combining the MCL1 inhibitor UMI-77 with either navitoclax (Figure 5d; Figure S6a) or A115 (Figure S6d) was examined. In combination, the addition of an MCL1 inhibitor does not enhance killing of senescent fibroblasts indicating that therapy-induced senescent fibroblasts are not dependent on MCL1 activity.

Finally, to assess the ability of navitoclax to target senescent fibroblasts in a more complex 3D environment, RFP-labelled D2A1 cell and MRC5-miR146a-GFP fibroblast 3D co-culture spheroids were treated for 72 hours with vehicle or doxorubicin and then for a further 72 hours in the presence or absence of navitoclax (Figure 5e). As previously 
demonstrated (Figure 3e), doxorubicin treatment results in elimination of D2A1 cells, as monitored by reduced RFP expression and reduction in cell viability within the spheroids, and is accompanied a concomitant increase in expression of the fibroblast GFP senescence reporter. Navitoclax has no impact on vehicle-treated 3D spheroids but in chemotherapytreated spheroids results in a further significant reduction in cell viability and a notable loss of GFP-expressing fibroblasts (Figure 5e).

\section{$\underline{\text { Resistance to senolytic drugs hampers efficacy in vivo }}$}

Encouraged by the efficacy of navitoclax in vitro, we investigated whether these agents were effective in eliminating chemotherapy-induced senescent cells in vivo to reduce their impact on metastatic tumour growth. BALB/c mice were treated with a course of chemotherapy or vehicle followed by 5 daily doses of navitoclax or vehicle and 3 days later inoculated intravenously with D2.OR tumour cells (Figure 6a). Consistent with previous data (Figure 1), on Day 77 there is significantly increased tumour burden in the chemotherapy-treated mice compared to vehicle-treated mice, monitored by IVIS imaging, however, there is no significant difference between groups which had, or had not, received subsequent navitoclax treatment (Figure 6b). On Day 91, when the first mice began to show signs of ill health, the experiment was terminated. In line with the Day 77 IVIS data (Figure 6b), chemotherapytreated mice have a significantly increased metastatic burden in the lungs, quantified by the numbers of metastatic lesions and percentage tumour area, and again, no significant effect of navitoclax treatment on vehicle-treated mice is observed (Figure 6c). However, it is notable that in comparison to chemotherapy-only treated mice, the mice receiving the combination therapy have a greater range of metastatic disease with a subgroup of mice exhibiting an increased metastatic burden and a subgroup of mice showing a reduced metastatic burden (Figure 6c). Strikingly equivalent results were obtained in NRG mice inoculated with human ZR-75-1 breast cancer cells (Figure 6d-f). As described previously (Figure 1; Figure S2), mice pre-treated with a course of chemotherapy develop significantly more liver metastases than vehicle treated mice (Figure 6e,f). Again, chemotherapy-treated 
mice that received a subsequent treatment with navitoclax show mixed results. There is no significant difference between the navitoclax and vehicle treated groups but navitoclax treatment results in a range of metastatic disease monitored by IVIS imaging and quantification of liver lesions.

In the study described in Figure 6a-f, mice were treated with chemotherapy and/or navitoclax prior to tumour cell inoculation to discriminate between any effect of treatment on the stromal and tumour compartments. However, since chemotherapy will always be administered after a patient's cancer diagnosis, it was important to test the combination of chemotherapy with navitoclax in a clinically relevant setting. In an initial experiment (Figure $6 \mathrm{~g})$, untreated BALB/c mice were inoculated intravenously with syngeneic D2A1 cells to allow tumour cells to colonise the lungs and placed into 4 groups with equal median tumour burden, based on IVIS imaging within 90 minutes of tumour cell inoculation (Figure 6h). 7 days post-inoculation, mice were treated with a course of chemotherapy or vehicle and, following a 3 day gap, then treated with 5 daily doses of navitoclax or vehicle (Figure $6 \mathrm{~g}$ ). Mice which did not receive chemotherapy show a median survival of 21 days.

Chemotherapy-treated mice have a significantly increased median survival of 33 days. Although there is no significant difference in the median survival of mice treated with or without navitoclax, 3 out of 8 mice receiving the combination treatment outlived mice receiving chemotherapy-alone, with one mouse exhibiting a complete remission (Figure 6i). Within the combination treatment group the longest surviving mice are those which had the lowest IVIS signal at the beginning of the navitoclax treatment (data not shown), suggesting that the response to second-line navitoclax treatment might be greater in mice with lower disease burden. To asses this, a spontaneous metastasis assay was performed (Figure 6j). Since the parental D2A1 cell line is weakly metastatic, mice were inoculated orthotopically with the metastatic D2A1-m2 subline (Jungwirth et al., 2018) and primary tumours were allowed develop for 12 days before treatment commenced. As expected, chemotherapy treatment significantly blunts primary tumour growth monitored by tumour volume and ex vivo tumour weight (Figure 6k) and significantly reduces metastatic burden in the lungs 
(Figure 6I). Navitoclax treatment, either alone or following chemotherapy treatment, has no effect on primary tumour growth. As a single agent navitoclax also has no effect on spontaneous metastasis. In the chemotherapy-treated groups, a subsequent course of navitoclax results in an additional $\sim 25 \%$ reduction in metastatic burden, however, this reduction is not significant (Figure 6l).

To investigate whether this lack of efficacy in limiting metastatic colonisation reflected an inability of navitoclax to clear senescent stromal cells in vivo, naive BALB/c mice were treated with a course of vehicle or chemotherapy followed by 4 daily doses of vehicle or navitoclax, and lungs were removed at necropsy 24 hours later (Day 24) (Figure 7a) for gene expression analysis. Lungs isolated from mice receiving chemotherapy alone show, as previously reported (Figure 2e; Figure S4), a significantly upregulated SASP expression, including increased expression of I/6, Lif, Cdkn1a (p21), Mmp3, Cc/2, Cc/7, Cxcl1 and Cxcl10 (Figure 7b; Figure S8; Table S1,S2), and PCA analysis reveals discrete clustering of the chemotherapy and vehicle groups (Figure S9a). Of note, when analysing these data together with the data shown in Figure $2 \mathrm{e}$, the vehicle and chemotherapy-treated samples from the independent experiments cluster together (Fig S9b), indicating a reproducible effect of chemotherapy treatment on the lung tissue despite having different experimental endpoints (Table S1,S2).

Lung tissue from mice treated with navitoclax as a monotherapy show no significant differences in expression across the 1344 target genes analysed on the two NanoString panels (Figure 7c; Figure S8). In addition, consistent with the lack of efficacy in metastasis assays, there is little indication that navitoclax reverses the effects of chemotherapy treatment, with only 7 genes within the gene expression panels showing a significant change in expression between the chemotherapy-treated groups receiving a subsequent course of navitoclax or vehicle (Figure 7c). Importantly, within these chemotherapy-treated groups, while subsequent navitoclax administration significantly reduces the expression of Lif and Ccl2 as well as the CCL2 receptor Ccr2, there is no significant reduction in expression of the senescence marker p21 (Cdkn1a) (Figure 7c) indicating that navitoclax is ineffective in 
removing senescent cells from chemotherapy-treated lung tissue in vivo. To confirm these findings, lung sections from the same mice were stained for $\mathrm{p} 21$. As previously reported (Figure 1b,c), very few p21-positive cells are detected in the lung tissue of vehicle-treated mice but their number significantly increases in lungs of chemotherapy-treated mice (Figure 7d). Consistent with the gene expression data, in chemotherapy-treated mice that received a subsequent course of navitoclax there is no change in the number of p21-positive cells (Figure 7d) confirming that, and in contrast to the in vitro findings (Figure 5), navitoclax is unable to eliminate p21-positive stromal cells in vivo. Moreover, $\mathrm{PyH} 2 \mathrm{Ax}$-positive cells are readily detected in chemotherapy-treated mice and, unexpectedly, this number significantly increases following a subsequent course of navitoclax treatment (Figure 7e). In light of the finding that $\mathrm{PyH} 2 \mathrm{Ax}$ staining peaks 3 days following chemotherapy treatment (Figure $2 \mathrm{~b}$ ), this suggests that in vivo navitoclax treatment prolongs the persistence of DNA-damaged senescent cells. Interestingly, two genes significantly upregulated in the chemotherapy plus navitoclax group are Bc/2/1, which encodes BCLxl, and Notch1 (Figure 7c). Notch signalling has been previously reported to induce senescence in a juxtacrine fashion (Hoare et al., 2016) and to exhibit anti-apoptotic properties through interactions with mitochondrial remodelling proteins, including BCL2 (Perumalsamy et al., 2010; Ye et al., 2012). Together this raises the possibility that upregulation of BCLxI and Notch1 could provide an in vivo resistance mechanism allowing DNA-damaged senescent stromal cells to survive navitoclax treatment. 


\section{Discussion}

The data presented here show that in multiple independent models of both immunecompetent and immune-compromised mice, pre-treatment with a clinically-relevant chemotherapy regimen of combined doxorubicin and cyclophosphamide followed by subsequent inoculation of tumour cells results in a significantly increased burden of metastatic disease. The use of the mouse D2.OR and human ER+ ZR-75-1 tumour cell lines, which exhibit a dormant or latent phenotype when inoculated into naive mice, has clinical relevance in the context of ER+ breast cancers which frequently exhibit a late relapse phenotype, developing metastatic disease years or decades after original diagnosis and treatment(Dittmer, 2017). In both the mouse models and in patients the presence of disseminated tumour cells in secondary sites results in prolonged exposure to the stromal microenvironment, which would be rendered a more pro-metastatic environment in patients receiving systemic chemotherapy as part of their treatment. Of note, in the ZR-75-1 model, although prior chemotherapy induces metastatic colonisation in multiple organs, the majority of metastatic burden is unexpectedly found in the liver, thus providing a much needed model of breast cancer liver metastasis.

Systemic delivery of chemotherapy causes widespread DNA damage in normal mouse tissues and increased accumulation in senescent cells, particularly senescent fibroblasts, together with their associated characteristic SASP. Cellular senescence is a survival program initiated in response to stress pathways that typically converge on DNA damage signalling (Fumagalli et al., 2014). Once a cell enters senescence the cell cycle is blocked permanently, but cells remain highly metabolically active. These chemotherapyinduced senescent cells, as monitored by expression of $\mathrm{p} 21$, persist in mouse tissues for several weeks following treatment before levels of p21 decline. However, the senescence phenotype is dynamic and evolves over time (Baker and Sedivy, 2013; Georgakopoulou et al., 2013), as exemplified in in vitro studies (Figure S4). After 3 weeks, although expression of markers such as p21 and the PmiR146a-GFP reporter decrease, longer-term senescence 
markers such as cytoplasmic lipofuscin granules accumulate; indicating a change in the expression of senescence markers, rather than a decline senescent cell number.

Whilst our 3D in vitro models support the hypothesis that increased inflammatory SASP signalling by stromal fibroblasts directly promotes tumour growth by increasing mitogenic signalling and tumour cell proliferation; there are other consequences that senescent cells could inflict on the microenvironment, contributing indirectly to metastatic colonisation. First, the senescent cell SASP can exhibit immune suppressive properties via the promotion of type-2 immune polarisation (Ruhland et al., 2016), resulting in reduced immune surveillance. Indeed, our cytokine array data reveal that amongst the most abundant chemokines secreted by senescent fibroblasts are macrophage inflammatory protein 1y (MIP1y, CCL9) and macrophage chemoattractant protein 1 (MCP1, CCL2), which is reflected in increased expression of $\mathrm{Cc} / 9$ and $\mathrm{C} c / 2$ in the chemotherapy-treated lung tissue. However, monitoring CD4+ and CD8+ cells in the lung tissue at five timepoints following a course of chemotherapy treatment revealed no differences in either population. Further, a profound pro-tumourigenic effect of chemotherapy treatment is observed in NRG mice, which lack any functional components of the immune system. Second, senescent cells also secrete pro-angiogenic factors such as VEGF (Coppé et al., 2008). Since establishing a blood supply has been shown to be required for dormant or latent tumours cells to re-initiate a proliferative program (Naumov et al., 2006), SASP-induced vascular remodelling could play a key role in senescence-promoted metastatic growth (Daenen et al., 2011). However, immunofluorescence staining failed to reveal any overt changes to the tissue vasculature following chemotherapy treatment, suggesting that this was also unlikely to be the driving factor for the observed increase in metastatic tumour growth.

The clinical importance of targeting both naturally-occurring and therapy-induced senescent cells has become increasingly clear over the past 5 years of research (Childs et al., 2017; Kirkland and Tchkonia, 2020). Seminal proof of principle experiments, involving the genetic elimination of cells expressing the senescence marker $p 16^{\text {Ink4a }}$, have demonstrated an alleviation of age and therapy-induced pathologies (Baker et al., 2016; 
Baker et al., 2011), including progression and metastasis in a PyMT-MMTV spontaneous mammary carcinoma model (Demaria et al., 2017). Experimental studies into the therapeutic targeting of senescent cells has focused on administration of the BLC2 mimetic navitoclax (ABT-263), the closely related inhibitor ABT-737 or the non-related senolytic combination of dasatinib and quercetin (D\&Q) which have been shown to eliminate or reduce the number of senescent cells in ageing mouse models and alleviative the ageing phenotype (Chang et al., 2016; Xu et al., 2018; Yosef et al., 2016). In our studies, D\&Q was not selective for senescent fibroblasts in vitro and had no effect in vivo (data not shown). By contrast, navitoclax or the BCLxI specific inhibitor A115 selectively eliminated chemotherapy-induced senescent fibroblasts in vitro. However, treatment with navitoclax, using dosing regimens shown to be effective in ageing mouse models (Chang et al., 2016), failed to eliminate chemotherapy-induced senescent cells in vivo and thus did not reduce tumour growth or extend survival in chemotherapy-treated mice. Whilst gene expression profiling provided some evidence of on-target effects of navitoclax in chemotherapy-treated mice, the number and magnitude of the changes were small. Moreover, mice receiving the combination treatment show a trend towards increased lung metastatic burden and increased levels of the DNA damage marker $\mathrm{PyH} 2 \mathrm{Ax}$, indicating the emergence of resistance mechanisms that prevent the elimination of senescent cells. Indeed, in the combination treated mice we observe significantly increased expression of $B c / 2 / 1$ (encoding $B C L x I)$. Our in vitro studies demonstrate that the efficacy of navitoclax in eliminating senescent fibroblasts is dependent on its ability to inhibit BCLxI, hence this upregulated expression could provide a senescent cell resistance mechanism in vivo. Indeed in in vivo models of non-Hodgkin's lymphoma, increased BCLxl levels have been reported to mediate resistance to ABT-737 (Mérino et al., 2012). Clinical trials of senolytic agents are ongoing for several pathologies (Ellison-Hughes, 2020; Kirkland and Tchkonia, 2020). However, studies including our own, have demonstrated the limitations and risks of using senolytic drugs (Sharma et al., 2020), highlighting the need for continued research in this area. Excitingly, anti-senescence therapies are now entering their second generation, with senolytics being developed which 
have improved tolerability and efficacy in vivo (González-Gualda et al., 2020; Guerrero et al., 2020), as well as chimeric antigen receptor (CAR) T cells targeting senescent cells (Amor et al., 2020).

In the context of using senolytic agents as part of breast cancer treatment, we have illustrated their potential role in ER+ breast cancer patients with disseminated latent disease. Although a late relapse phenotype is much less common in hormone receptor negative breast cancers and these cancers frequently respond well to chemotherapy, there remains a significant proportion of patients with chemoresistant residual disease. In both these scenarios, the data presented here highlight the potential negative consequences of chemotherapy treatment in which chemotherapy-mediated damage and induction of senescence in normal tissue, paradoxically, has the potential to increase the risk of recurrence. In conclusion, targeting senescent fibroblasts represents an exciting clinical opportunity to minimise these negative side effects of chemotherapy, however, further development of senolytic strategies is required to unlock their full potential. 


\section{Experimental procedures}

\section{$\underline{\text { Reagents and cells }}$}

RTqPCR probes are listed in Table S3. Antibodies, and the dilutions used, are detailed in Table S4. MRC5 and IMR90 fibroblasts were purchased from ATCC in 2009 and 2016, respectively. NIH-3T3 and ZR-75-1 cells were from Isacke laboratory stocks. D2A1 and D2.OR cells were from Ann Chambers' laboratory stocks (Morris et al., 1994). The generation of the metastatic D2A1-m2 subline has been described previously (Jungwirth et al., 2018). All cells were used within ten passages after resuscitation and were routinely subject to mycoplasma testing. ZR-75-1 cells were short tandem repeat tested every 4 months (Stem Elite ID System, Promega). Doxorubicin (S1208), docetaxel (S1148), navitoclax (S1001), A-1155463 (S7800), venetoclax (S8048) and UMI-77(S7531) were purchased from Selleckchem. Cyclophosphamide monohydrate was purchased from SigmaAldrich (C0768).

Lentiviral transfection of tumour cells with RFP, luciferase and mCherry: As indicated, tumour cells were transduced with lentiviral expression particles containing either: (1) a firefly luciferase gene with a blasticidin-resistance gene (cells denoted -Luc) (Amsbio, LVP326); (2) a firefly luciferase gene, with an mCherry encoding gene (cells denoted mChLuc2) (Genescript, Sall-IRES-Luc2-XbalXhol_PGK-H2BmCherry); or (3) an RFP encoding gene (cells denoted -RFP) (lentiviral vector pDEST/pHIV-H2BmRFP-rfa_verB, a gift from Matthew Smalley, University of Cardiff). mCherry+/RFP+ cells were selected by fluorescence-activated cell sorting (FACS). Luciferase only transduced cells were enriched by culturing the cells in DMEM with 10\% FBS containing blasticidin for 2-3 passages.

Lentiviral transfection of fibroblasts with PmiR146a-GFP plasmid: The miR146a-GFP plasmid $(35 \mu \mathrm{g})($ Kang et al., 2015) was combined with packaging vectors pMD2.G (11 $\mu \mathrm{g})$ and psPAX2 $(25.6 \mu \mathrm{g})$ in $18 \mathrm{~mL}$ Optimem medium containing $216 \mu \mathrm{L}$ Lipofectamine-2000, and incubated at room temperature for 20 minutes. Lentivirus containing medium from HEK293T cells was harvested at 24 and 48 hours, centrifuged at $300 \mathrm{~g}$ and filtered through a $0.2 \mu \mathrm{m}$ syringe filter before use for cell transfections. Fibroblasts were seeded in $25 \mathrm{~cm}^{2}$ 
flasks in medium containing miR146a-GFP lentiviral particles and $4 \mu \mathrm{g} \mathrm{mL}{ }^{-1}$ polybrene and for incubated 24 hours. Virus containing medium was replenished after 24 hours.

Transfected fibroblasts were then maintained in DMEM with 10\% FBS containing puromycin for 2-3 passages.

\section{$\underline{\text { In vivo procedures }}$}

All animal work was carried out under UK Home Office Project Licenses 70/7413 and P6AB1448A granted under the Animals (Scientific Procedures) Act 1986 (Establishment Licence, X702B0E74 70/2902) and was approved by the 'Animal Welfare and Ethical Review Body' at The Institute of Cancer Research (ICR). All mice used were female and aged between 6-9 weeks and 18-25 $\mathrm{g}$ in weight at the beginning of an experiment.

Syngeneic studies were carried out in BALB/c mice. Human cell line studies were carried out in NOD RAG gamma (NRG) mice (NOD-Rag $1^{-/-} \mathrm{IL} 2 \mathrm{rg}^{-/-}$), implanted with 90 day, sustained release $0.36 \mathrm{mg} 17 \beta$-oestrodiol pellets (Innovative Research of America, NE-121) 4-5 days prior to tumour cell implantation. All mice were housed in individually ventilated cages, monitored daily by ICR Biological Services Unit staff and had food and water ad libitum. In all cases, experiments were terminated if the primary tumour reached a maximum allowable diameter of $17 \mathrm{~mm}$, if thoracic IVIS signal exceeded $1 \times 10^{9}$ photons per second or if a mouse showed signs of ill health. For non-tumour bearing mice, mice were randomised into groups based on body weight. For experimental and spontaneous metastasis experiments, mice were randomised prior to drug administration based on IVIS signal or tumour volume, respectively.

Chemotherapy treatment of mice: Mice were dosed via $100 \mu \mathrm{L}$ intraperitoneal injection with a combination of doxorubicin (2.3-2.7 $\mathrm{mg} \mathrm{kg}^{-1}$, Selleckchem S1208) and cyclophosphamide (40-44 $\mathrm{mg} \mathrm{kg}^{-1}$, Sigma 50850) in 0.9\% NaCl. Doxorubicin and cyclophosphamide were stored separately at a $2 \mathrm{X}$ concentration $(1.7 \mathrm{mM}$ and $57 \mathrm{mM}$, respectively) and were mixed at a 1:1 ratio immediately prior to injection. Vehicle-treated mice were injected intraperitoneally with $100 \mu \mathrm{L} 0.9 \% \mathrm{NaCl}$. 
Navitoclax treatment of mice: Navitoclax was stored as aliquots of $100 \mathrm{mM}$ solution in DMSO at $-80^{\circ} \mathrm{C}$. For each dose one aliquot was thawed and diluted in corn oil to a final concentration of $5 \mathrm{mM}$ (5\% DMSO final concentration). Vehicle-treated mice were treated with a $5 \%$ solution of DMSO in corn oil. Mice were dosed via oral gavage using a $200 \mu \mathrm{L}$ volume.

Spontaneous metastasis assays: $2 \times 10^{5} \mathrm{D} 2 \mathrm{~A} 1-\mathrm{m} 2$ cells were injected orthotopically into the $4^{\text {th }}$ mammary fat pad under general anaesthesia. Tumour growth was measured twice a week using callipers up to a maximum diameter of $17 \mathrm{~mm}$. Tumour volume was calculated as $0.5236 \times[(\text { width }+ \text { length }) / 2]^{\wedge} 3$. At endpoint tumours were excised and weighed.

Intravenous inoculation: $5 \times 10^{5} \mathrm{D} 2 \mathrm{~A} 1,1 \times 10^{6} \mathrm{D} 2 . \mathrm{OR}$ or $2 \times 10^{6} \mathrm{ZR}-75-1$ cells (transfected with either -Luc or -mChLuc2 vectors) were injected into the lateral tail vein of mice. Metastatic growth was monitored by repeated IVIS imaging starting at $\sim 90$ minutes following inoculation. At endpoint lungs were excised and, where indicated, IVIS imaged ex vivo.

IVIS imaging: Mice were injected intraperitoneally with $150 \mathrm{mg} \mathrm{kg}^{-1}$ D-luciferin (Caliper Life Sciences) in $100 \mu \mathrm{L}$ and mice imaged in vivo using an IVIS imaging chamber (IVIS Illumina II). Luminescence measurements (photons per second per $\mathrm{cm}^{2}$ ) were acquired over 1 minute and analysed using the Living Image software (PerkinElmer) using a constant size region of interest over the tissues. Alternatively, $>15$ minutes after D-luciferin injection, dissected lungs and/or livers were imaged ex vivo.

Quantification of metastatic burden and immunostaining: Tissues were formalin-fixed and paraffin-embedded (FFPE). 3-4 $\mu \mathrm{m}$ lung, liver or bone FFPE sections were cut, dewaxed in xylene, re-hydrated through ethanol washes and stained with haematoxylin and eosin (H\&E). Slides were scanned using Hamamatsu microscope with a NanoZoomer XR camera using the $20 x$ objective and file names blinded. For quantifying tumour burden, total number of individual nodules was counted manually in 1-3 sections approximately $150 \mu \mathrm{m}$ apart, per tissue. Lung metastatic area was quantified as the mean size of the nodules or 
percentage of the area of the metastatic nodules normalised to the total lung area.

Alternatively, sections were subjected to high-temperature antigen retrieval, blocked using avidin/biotin, before incubation with primary antibodies. Immunohistochemical detection was achieved with the VectaStain ABC system. Stained sections were scanned as described above and file names blinded. p21 or $\mathrm{P}-\mathrm{yH} 2 \mathrm{Ax}$ positive cells were quantified manually from $\geq 6$ randomly selected $0.1 \mathrm{~mm}^{2}$ fields of view per lung section, avoiding regions containing bronchi and bronchioles. Alternatively, FFPE sections of mouse lungs were stained with p21 alongside aSMA, endomucin or EpCAM and DAPI, then incubated with the appropriate secondary antibody conjugated to fluorophores. Fluorescent stained sections were scanned as described above. p21 positive cells were counted across the whole section. p21 positive cells were then scored for positivity of aSMA, endomucin or EpCAM. Representative higher power images were collected on a Leica SP8 microscope using a 40X oil immersion objective.

RNA extraction from tissue: Mice were sacrificed 7 or 10 days after the final chemotherapy or vehicle treatment. The lungs were excised and the left lobe was fixed and processed for immunohistochemical staining as described above. The right lobes were immediately snap-frozen in liquid nitrogen in a cryovial and then stored at $-80^{\circ} \mathrm{C}$. To extract RNA, a 20 mg chunk of frozen lung tissue was collected using a scalpel and placed in $1 \mathrm{~mL}$ RLT buffer (Qiagen) with 1:100 $\beta$-mercaptoethanol in a Precellys silicone bead dissociation tube (Bertin-Corp). Tissue were dissociated by rigorous vortexing for 1 minute using the Precellys 24 Tissue Homogenizer and lysates immediately frozen at $-80^{\circ} \mathrm{C}$. RNA extraction was carried out using the Qiagen RNeasy kit according to the manufacturer's protocol.

\section{$\underline{\text { In vitro studies }}$}

RTqPCR: RNA was isolated using the Qiagen RNeasy kit and cDNA was generated by using the QuantiTect reverse transcription kit (Qiagen) according to the manufacturer's instructions. RTqPCR was performed with human or mouse Taqman Gene Expression Assay probes on an Applied Biosciences QuantStudio6 Flex Real-time PCR machine and 
relative quantification was performed using QuantStudio Real-time PCR software. Each reaction was performed in triplicate. Relative expression levels were normalised to B2m/B2M, Gapdh/GAPDH, or Tmem199/TMEM199 endogenous controls, and $\geq 2$ controls were used in each experiment. In control 3T3 fibroblast RNA samples, the Mmp3 and I/1a probes did not amplify during the run. Consequently, $R Q$ values were calculated using an assumed CT value of 40 for the control samples, based on the maximum number of cycles used. Confidence intervals were set at $95 \%$ for all assays.

NanoString gene expression analysis: RNA was extracted from frozen lung tissue as described above. 80 ng RNAwas hybridised with the Mouse PanCancer Immune panel or Mouse PanCancer Pathways panel and processed using the nCounter SPRINT Profiler (NanoString) following the manufacturer's instructions. Raw NanoString data was preprocessed using R package NanoStringNorm (v1.2.1) ( Waggott et al., 2012) and further normalised using voom (TMM normalisation) followed by differential gene expression analysis with R package limma (v3.40.6) (Ritchie et al., 2015) Genes with absolute $\log _{2}$ fold change $\geq 0.585$ and FDR adjusted $p$ value $<0.05$ were considered significantly different. Principle component analysis (PCA) was performed using R package FactoMineR(v2.3). All analyses were performed in R statistical programming language (v3.6.14). Visualisations were generated using in-house plotting libraries.

Chemotherapy induction of senescence: Fibroblasts were cultured in DMEM with $10 \%$ FBS in $75 \mathrm{~cm}^{2}$ flasks to $\sim 75 \%$ confluence, then medium was replaced with DMEM with 10\% FBS containing chemotherapeutic agents. Fibroblasts were incubated in medium containing the drug for 24 hours, washed twice with PBS and then cultured in fresh DMEM with $10 \%$ FBS. Senescent fibroblasts were maintained in the same flasks for up to 6 months without further passage, growth medium was replaced weekly with fresh DMEM plus $10 \%$ FBS.

Senescent cell imaging: Fibroblasts induced senescence as described above were seeded in 6 well plates at $80-90 \%$ confluency $7-21$ days following drug withdrawal and incubated for 24 hours before staining. Control fibroblasts were seeded into a 6-well plate 
and cultured for 2-3 days until they matched the confluency of the senescent fibroblasts. Fibroblasts were washed with PBS, fixed for 10 minutes with $2 \%$ paraformaldehyde and $0.2 \%$ glutaraldehyde, washed twice with PBS and $1.5 \mathrm{~mL}$ staining solution (SA $\beta-$ Gal histochemical staining kit, Sigma CS0030) containing X-Gal was added. Plates were sealed with Parafilm and incubated at $37^{\circ} \mathrm{C}$ in a non-humidified incubator at atmospheric $\mathrm{CO}_{2}$ concentration. After 24 hours the cells were washed with PBS and imaged at 100x magnification. For live cell imaging of GFP signal, cells were imaged using either the EVOS fluorescence microscope or the Incucyte live cell imaging fluorescence microscope using Excitation 470/Emission 525 wavelengths.

Conditioned media (CM): CM was collected from senescent fibroblasts 7-30 days following chemotherapy withdrawal. Senescent or control fibroblasts were washed twice and then cultured in serum-free DMEM. After 24 hours, CM was collected, centrifuged at $300 \mathrm{~g}$, filtered through a $0.2 \mu \mathrm{m}$ pore filter and used fresh or stored at $-80^{\circ} \mathrm{C}$ for future use. $\mathrm{CM}$ was used within 6 months of freezing and was filtered post-thawing.

Cytokine arrays: CM was freshly collected as described above from senescent human and mouse fibroblasts or from control fibroblasts. CM was collected independently from two flasks per condition, and $100 \mu \mathrm{L}$ added to duplicate RayBiotech G2000 array slides. Wash and incubation steps were performed according to the manufacturer's protocol. Fluorescence signal detection at $532 \mathrm{~nm}$ was performed on a GenePix 4000B array scanner. Median fluorescence intensity (MFI) was quantified using ImageJ software. Fold changes were calculated by dividing the mean MFI for the two senescent CM replicates by the mean MFI for the two control replicates for each fibroblast type.

Phosphoprotein arrays: Tumour cells were seeded into ultra-low attachment Ubottom 96-well plates in DMEM with $2 \%$ FBS at density of 12,500 cells per well and incubated for 48 hours. 192 spheroids were divided into 2 groups of 96 and placed into 15 $\mathrm{mL}$ Falcon tubes, washed once with PBS and then incubated in $12 \mathrm{~mL}$ serum-free DMEM for 2 hours at $37^{\circ} \mathrm{C}$ on a roller. Spheroids were centrifuged at $260 \mathrm{~g}$, medium removed and replaced with $10 \mathrm{~mL}$ of control or senescent fibroblast $\mathrm{CM}$ and incubated for 30 minutes at 
$37^{\circ} \mathrm{C}$ on a roller. Spheroids were then washed $2 \mathrm{X}$ in $10 \mathrm{~mL}$ ice-cold PBS then lysed in 100 $\mu \mathrm{L}$ Cell Signalling Technologies lysis buffer containing $1 \mathrm{mM}$ PMSF. Cell lysates were sonicated in an ice-cold water bath for 10 minutes, frozen to $-80^{\circ} \mathrm{C}$ for 30 minutes, thawed, centrifuged at $16,000 \mathrm{~g}$ for 10 minutes at $4^{\circ} \mathrm{C}$, the supernatant was transferred to a fresh tube on ice and the pellet discarded. Protein concentration was measured using the Millipore Direct Detect Spectrometer according to the manufacturer's protocol. Protein samples were diluted in array blocking buffer and $150 \mu \mathrm{L}$ was added to the Pathscan antibody-array slides (Cell Signalling Technologies \#7982). Wash and incubation steps, and sample detection using a chemi-luminescent HRP substrate were performed according to the manufacturer's protocol. Luminescence was imaged using the Odyssey Fc Imaging System. Median fluorescence intensity (MFI) was quantified using ImageJ software. Fold changes were calculated by dividing the mean MFI for the two technical replicate spots for each target in the senescent CM treated spheroids by the mean MFI for the two technical replicate spots for each target in the control CM treated spheroids.

Co-culture spheroid assays: 1,000 tumour cells and 1,000 fibroblasts were coseeded into ultra-low attachment U-bottom 96-well plates and incubated for 24 hours. Spheroids were treated with doxorubicin $(0-0.85 \mu \mathrm{M})$ and incubated for a further 72 hours before imaging on an EVOS microscope. Alternatively, 5,000 tumour cells and 5,000 fibroblasts were co-seeded, incubated for 24 hours before addition of $0.17 \mu \mathrm{M}$ doxorubicin or vehicle. 72 hours later, spheroids were treated with $4.5 \mu \mathrm{M}$ navitoclax or vehicle and incubated for a further 72 hours before being imaged and assayed by CellTiter-Glo.

Senolytic dose response assays: Senescent fibroblasts were seeded into 96-well or 24-well plates for CellTiter-Glo or crystal violet analysis, respectively. Senescent fibroblasts were seeded at $\sim 95 \%$ confluence in parallel with control fibroblasts at $\sim 70 \%$ confluence and incubated for 24 hours prior to treatment with a senolytic agents, alone or in combination, as indicated for 72 hours before assaying cell viability by CellTiter-Glo or staining with $0.5 \%$ crystal violet in methanol. 


\section{$\underline{\text { Statistics }}$}

Statistics were performed using GraphPad Prism 8. All data is represented as the mean value \pm standard error of the mean. Comparisons between two groups were made using two-tailed, unpaired Student's $t$-tests. If there was a significant difference between the standard deviations of the two groups, then Welch's correction was applied. If the analysis did not pass the Shapiro-Wilk normality test, then groups were analysed by Mann-Whitney U-test. If more than 2 groups were compared One-way ANOVA analysis was performed with Dunnett's correction for multiple comparisons. If a significant difference between the standard deviations of the means was measured then the Brown-Forsythe and Welch correction was applied. If data did not pass the Shapiro-Wilk normality test then a Kruskal-Wallis ANOVA was performed with Dunn's correction for multiple comparisons. ${ }^{*}, p$ $<0.05 ;{ }^{* *} p<0.01 ;{ }^{* * *}, p<0.001 ;$ ns, not significant.

\section{Supplemental Information}

Supplemental Information includes 9 figures and 4 tables

\section{Acknowledgements}

This work was funded as part of Programme Funding to the Breast Cancer Now Toby Robins Research Centre (CMI) and a Chairman's ICR PhD studentship (DP). We acknowledge NHS funding to the NIHR Biomedical Research Centre at the Royal Marsden and the ICR. We would like to thank Steve Elledge for the PmiR146a-GFP plasmid, David Vicente and Sarah Ash for experimental contribution, the ICR Biological Services Unit and Dr Naomi Guppy and her team in the Breast Cancer Now Toby Robins Research Centre Nina Barough Pathology Core Faculty for histopathology support.

\section{Author Contributions}

Conceptualization (DWP, LO'L, CMI) 
Investigation (DWP, SH, DR, RB, LO'L, CMI)

Writing - Review \& Editing (DWP, CMI with input from all other authors)

Funding Acquisition (SH, CMI)

\section{Declaration of interests}

The authors have no financial or non-financial competing interests to declare 


\section{References}

Acosta, J.C., Banito, A., Wuestefeld, T., Georgilis, A., Janich, P., Morton, J.P., Athineos, D., Kang, T.W., Lasitschka, F., Andrulis, M., et al. (2013). A complex secretory program orchestrated by the inflammasome controls paracrine senescence. Nat Cell Biol 15, 978-990.

Alspach, E., Flanagan, K.C., Luo, X., Ruhland, M.K., Huang, H., Pazolli, E., Donlin, M.J., Marsh, T., Piwnica-Worms, D., Monahan, J., et al. (2014). p38MAPK plays a crucial role in stromal-mediated tumorigenesis. Cancer Discov 4, 716-729.

Amor, C., Feucht, J., Leibold, J., Ho, Y.-J., Zhu, C., Alonso-Curbelo, D., Mansilla-Soto, J., Boyer, J.A., Li, X., Giavridis, T., et al. (2020). Senolytic CAR T cells reverse senescence-associated pathologies. Nature 583, 127-132.

Ashenden, M., van Weverwijk, A., Murugaesu, N., Fearns, A., Campbell, J., Gao, Q., Iravani, M., and Isacke, C.M. (2017). An <em>In Vivo</em> Functional Screen Identifies JNK Signaling As a Modulator of Chemotherapeutic Response in Breast Cancer. Molecular Cancer Therapeutics 16, 1967-1978.

Azazmeh, N., Assouline, B., Winter, E., Ruppo, S., Nevo, Y., Maly, A., Meir, K., Witkiewicz, A.K., Cohen, J., Rizou, S.V., et al. (2020). Chronic expression of p16INK4a in the epidermis induces Wnt-mediated hyperplasia and promotes tumor initiation. Nature Communications 11, 2711.

Baker, D.J., Childs, B.G., Durik, M., Wijers, M.E., Sieben, C.J., Zhong, J., A. Saltness, R., Jeganathan, K.B., Verzosa, G.C., Pezeshki, A., et al. (2016). Naturally occurring p16Ink4a-positive cells shorten healthy lifespan. Nature 530, 184.

Baker, D.J., Perez-Terzic, C., Jin, F., Pitel, K.S., Niederlander, N.J., Jeganathan, K., Yamada, S., Reyes, S., Rowe, L., Hiddinga, H.J., et al. (2008). Opposing roles for p16Ink4a and p19Arf in senescence and ageing caused by BubR1 insufficiency. Nat Cell Biol 10, 825-836.

Baker, D.J., and Sedivy, J.M. (2013). Probing the depths of cellular senescence. The Journal of Cell Biology 202, 11-13. 
Baker, D.J., Wijshake, T., Tchkonia, T., LeBrasseur, N.K., Childs, B.G., van de Sluis, B., Kirkland, J.L., and van Deursen, J.M. (2011). Clearance of p16Ink4a-positive senescent cells delays ageing-associated disorders. Nature 479, 232-236.

Barkan, D., El Touny, L.H., Michalowski, A.M., Smith, J.A., Chu, I., Davis, A.S., Webster, J.D., Hoover, S., Simpson, R.M., Gauldie, J., et al. (2010). Metastatic Growth from Dormant Cells Induced by a Col-I-Enriched Fibrotic Environment. Cancer Research 70, 5706-5716.

Barkan, D., Kleinman, H., Simmons, J.L., Asmussen, H., Kamaraju, A.K., Hoenorhoff, M.J., Liu, Z.-y., Costes, S.V., Cho, E.H., Lockett, S., et al. (2008). Inhibition of Metastatic Outgrowth From Single Dormant Tumor Cells by Targeting the Cytoskeleton. Cancer research 68, 6241-6250.

Brunk, U.T., and Terman, A. (2002). Lipofuscin: Mechanisms of age-related accumulation and influence on cell function. Free Radical Biology and Medicine 33, 611-619.

Cai, Y., Zhou, H., Zhu, Y., Sun, Q., Ji, Y., Xue, A., Wang, Y., Chen, W., Yu, X., Wang, L., et al. (2020). Elimination of senescent cells by $\beta$-galactosidase-targeted prodrug attenuates inflammation and restores physical function in aged mice. Cell Research 30, 574-589.

Chang, J., Wang, Y., Shao, L., Laberge, R.M., Demaria, M., Campisi, J., Janakiraman, K., Sharpless, N.E., Ding, S., Feng, W., et al. (2016). Clearance of senescent cells by ABT263 rejuvenates aged hematopoietic stem cells in mice. Nat Med 22, 78-83.

Childs, B.G., Baker, D.J., Wijshake, T., Conover, C.A., Campisi, J., and van Deursen, J.M. (2016). Senescent intimal foam cells are deleterious at all stages of atherosclerosis. Science $354,472-477$.

Childs, B.G., Gluscevic, M., Baker, D.J., Laberge, R.-M., Marquess, D., Dananberg, J., and van Deursen, J.M. (2017). Senescent cells: an emerging target for diseases of ageing. Nature Reviews Drug Discovery 16, 718-735.

Coppé, J.-P., Patil, C.K., Rodier, F., Sun, Y., Muñoz, D.P., Goldstein, J., Nelson, P.S., Desprez, P.-Y., and Campisi, J. (2008). Senescence-associated secretory 
phenotypes reveal cell-nonautonomous functions of oncogenic RAS and the p53 tumor suppressor. PLoS biology 6, 2853-2868.

Cox, T.R., and Erler, J.T. (2014). Molecular Pathways: Connecting Fibrosis and Solid Tumor Metastasis. Clinical Cancer Research 20, 3637-3643.

Daenen, L.G., Roodhart, J.M., van Amersfoort, M., Dehnad, M., Roessingh, W., Ulfman, L.H., Derksen, P.W., and Voest, E.E. (2011). Chemotherapy enhances metastasis formation via VEGFR-1-expressing endothelial cells. Cancer Res 71, 6976-6985. de Carné Trécesson, S., Guillemin, Y., Bélanger, A., Bernard, A.-C., Preisser, L., Ravon, E., Gamelin, E., Juin, P., Barré, B., and Coqueret, O. (2011). Escape from p21mediated Oncogene-induced Senescence Leads to Cell Dedifferentiation and Dependence on Anti-apoptotic Bcl-xL and MCL1 Proteins. The Journal of Biological Chemistry 286, 12825-12838.

Demaria, M., O'Leary, M.N., Chang, J., Shao, L., Liu, S., Alimirah, F., Koenig, K., Le, C., Mitin, N., Deal, A.M., et al. (2017). Cellular Senescence Promotes Adverse Effects of Chemotherapy and Cancer Relapse. Cancer Discov 7, 165-176.

Dittmer, J. (2017). Mechanisms governing metastatic dormancy in breast cancer. Seminars in Cancer Biology 44, 72-82.

Dörr, J.R., Yu, Y., Milanovic, M., Beuster, G., Zasada, C., Dabritz, J.H., Lisec, J., Lenze, D., Gerhardt, A., Schleicher, K., et al. (2013). Synthetic lethal metabolic targeting of cellular senescence in cancer therapy. Nature 501, 421-425.

Eichhorn, J.M., Alford, S.E., Sakurikar, N., and Chambers, T.C. (2014). Molecular analysis of functional redundancy among anti-apoptotic $\mathrm{Bcl}-2$ proteins and its role in cancer cell survival. Experimental cell research 322, 415-424.

Ellison-Hughes, G.M. (2020). First evidence that senolytics are effective at decreasing senescent cells in humans. EBioMedicine 56, 102473.

Fumagalli, M., Rossiello, F., Mondello, C., and D'Adda Di Fagagna, F. (2014). Stable cellular senescence is associated with persistent DDR activation. PLoS ONE 9, 44-46. 
Gawrzak, S., Rinaldi, L., Gregorio, S., Arenas, E.J., Salvador, F., Urosevic, J., FiguerasPuig, C., Rojo, F., del Barco Barrantes, I., Cejalvo, J.M., et al. (2018). MSK1 regulates luminal cell differentiation and metastatic dormancy in ER+ breast cancer. Nature Cell Biology 20, 211-221.

Georgakopoulou, E.A., Tsimaratou, K., Evangelou, K., Fernandez Marcos, P.J., Zoumpourlis, V., Trougakos, I.P., Kletsas, D., Bartek, J., Serrano, M., and Gorgoulis, V.G. (2013). Specific lipofuscin staining as a novel biomarker to detect replicative and stress-induced senescence. A method applicable in cryo-preserved and archival tissues. Aging 5, 37-50.

González-Gualda, E., Pàez-Ribes, M., Lozano-Torres, B., Macias, D., Wilson, J.R., 3rd, González-López, C., Ou, H.L., Mirón-Barroso, S., Zhang, Z., Lérida-Viso, A., et al. (2020). Galacto-conjugation of Navitoclax as an efficient strategy to increase senolytic specificity and reduce platelet toxicity. Aging Cell 19, e13142.

Gonzalez-Meljem, J.M., Apps, J.R., Fraser, H.C., and Martinez-Barbera, J.P. (2018). Paracrine roles of cellular senescence in promoting tumourigenesis. British Journal of Cancer 118, 1283-1288.

Guerrero, A., Guiho, R., Herranz, N., Uren, A., Withers, D.J., Martínez-Barbera, J.P., Tietze, L.F., and Gil, J. (2020). Galactose-modified duocarmycin prodrugs as senolytics. Aging Cell 19, e13133.

Guida, J.L., Agurs-Collins, T., Ahles, T.A., Campisi, J., Dale, W., Demark-Wahnefried, W., Dietrich, J., Fuldner, R., Gallicchio, L., Green, P.A., et al. (2020). Strategies to Prevent or Remediate Cancer and Treatment-Related Aging. Journal of the National Cancer Institute.

Hoare, M., Ito, Y., Kang, T.-W., Weekes, M.P., Matheson, N.J., Patten, D.A., Shetty, S., Parry, A.J., Menon, S., Salama, R., et al. (2016). NOTCH1 mediates a switch between two distinct secretomes during senescence. Nature Cell Biology 18, 979. Hodge, D.R., Hurt, E.M., and Farrar, W.L. (2005). The role of IL-6 and STAT3 in inflammation and cancer. Eur J Cancer 41, 2502-2512. 
Johansen, P.B. (1981). Doxorubicin pharmacokinetics after intravenous and intraperitoneal administration in the nude mouse. Cancer Chemotherapy and Pharmacology 5, 267-270.

Jungwirth, U., van Weverwijk, A., Melake, M.J., Chambers, A.F., Gao, Q., Fivaz, M., and Isacke, C.M. (2018). Generation and characterisation of two D2A1 mammary cancer sublines to model spontaneous and experimental metastasis in a syngeneic BALB/c host. Disease Models \&amp; Mechanisms 11.

Jurk, D., Wang, C., Miwa, S., Maddick, M., Korolchuk, V., Tsolou, A., Gonos, E.S., Thrasivoulou, C., Jill, S.M., Cameron, K., et al. (2012). Postmitotic neurons develop a p21-dependent senescence-like phenotype driven by a DNA damage response. Aging Cell 11, 996-1004.

Kang, C., Xu, Q., Martin, T.D., Li, M.Z., Demaria, M., Aron, L., Lu, T., Yankner, B.A., Campisi, J., and Elledge, S.J. (2015). The DNA damage response induces inflammation and senescence by inhibiting autophagy of GATA4. Science (New York, NY) 349, aaa5612-aaa5612.

Kirkland, J.L., and Tchkonia, T. (2020). Senolytic Drugs: From Discovery to Translation. Journal of internal medicine.

Kobayashi, Y., Kadoya, T., Amioka, A., Hanaki, H., Sasada, S., Masumoto, N., Yamamoto, H., Arihiro, K., Kikuchi, A., and Okada, M. (2018). Wnt5a-induced cell migration is associated with the aggressiveness of estrogen receptor-positive breast cancer. Oncotarget 9, 20979-20992.

Krtolica, a., Parrinello, S., Lockett, S., Desprez, P.Y., and Campisi, J. (2001). Senescent fibroblasts promote epithelial cell growth and tumorigenesis: a link between cancer and aging. Proceedings of the National Academy of Sciences of the United States of America 98, 12072-12077.

Li, X., Yang, Q., Yu, H., Wu, L., Zhao, Y., Zhang, C., Yue, X., Liu, Z., Wu, H., Haffty, B.G., et al. (2014). LIF promotes tumorigenesis and metastasis of breast cancer through the AKT-mTOR pathway. Oncotarget 5, 788-801. 
Malaquin, N., Vancayseele, A., Gilbert, S., Antenor-Habazac, L., Olivier, M.A., Ait Ali Brahem, Z., Saad, F., Delouya, G., and Rodier, F. (2020). DNA Damage- But Not Enzalutamide-Induced Senescence in Prostate Cancer Promotes Senolytic Bcl-xL Inhibitor Sensitivity. Cells 9.

Mérino, D., Khaw, S.L., Glaser, S.P., Anderson, D.J., Belmont, L.D., Wong, C., Yue, P., Robati, M., Phipson, B., Fairlie, W.D., et al. (2012). Bcl-2, Bcl-xL, and Bcl-w are not equivalent targets of ABT-737 and navitoclax (ABT-263) in lymphoid and leukemic cells. Blood 119, 5807-5816.

Milanovic, M., Fan, D.N.Y., Belenki, D., Dabritz, J.H.M., Zhao, Z., Yu, Y., Dorr, J.R., Dimitrova, L., Lenze, D., Monteiro Barbosa, I.A., et al. (2018). Senescenceassociated reprogramming promotes cancer stemness. Nature 553, 96-100.

Morris, V.L., Koop, S., MacDonald, I.C., Schmidt, E.E., Grattan, M., Percy, D., Chambers, A.F., and Groom, A.C. (1994). Mammary carcinoma cell lines of high and low metastatic potential differ not in extravasation but in subsequent migration and growth. Clinical \& Experimental Metastasis 12, 357-367.

Naumov, G.N., Akslen, L.A., and Folkman, J. (2006). Role of Angiogenesis in Human Tumor Dormancy: Animal Models of the Angiogenic Switch. Cell Cycle 5, 1779-1787.

Niwa, H., Ogawa, K., Shimosato, D., and Adachi, K. (2009). A parallel circuit of LIF signalling pathways maintains pluripotency of mouse ES cells. Nature 460, 118-122.

Oeffinger, K.C., Mertens A.C. , Sklar C.A., Kawashima T., Hudson M.M., Meadows A.T., Friedman D.L., Marina N., Hobbie W.C.P., N.S., K.-L., et al. (2006). Chronic health conditions in adult survivors of childhood cancer. N Engl J Med 355, 1572-1582.

Park, S.I., Liao, J., Berry, J.E., Li, X., Koh, A.J., Michalski, M.E., Eber, M.R., Soki, F.N., Sadler, D., Sud, H., et al. (2012). Cyclophosphamide creates a receptive microenvironment for prostate cancer skeletal metastasis. Cancer Research 72, 2522-2532. 
Passos, J.F., Nelson, G., Wang, C., Richter, T., Simillion, C., Proctor, C.J., Miwa, S., Olijslagers, S., Hallinan, J., Wipat, A., et al. (2010). Feedback between p21 and reactive oxygen production is necessary for cell senescence. Mol Syst Biol 6, 347.

Pein, M., Insua-Rodríguez, J., Hongu, T., Riedel, A., Meier, J., Wiedmann, L., Decker, K., Essers, M.A.G., Sinn, H.-P., Spaich, S., et al. (2020). Metastasis-initiating cells induce and exploit a fibroblast niche to fuel malignant colonization of the lungs. Nature Communications 11, 1494.

Perrott, K.M., Wiley, C.D., Desprez, P.Y., and Campisi, J. (2017). Apigenin suppresses the senescence-associated secretory phenotype and paracrine effects on breast cancer cells. Geroscience 39, 161-173.

Perumalsamy, L.R., Nagala, M., and Sarin, A. (2010). Notch-activated signaling cascade interacts with mitochondrial remodeling proteins to regulate cell survival. Proceedings of the National Academy of Sciences 107, 6882-6887.

Ritchie, M.E., Phipson, B., Wu, D., Hu, Y., Law, C.W., Shi, W., and Smyth, G.K. (2015). limma powers differential expression analyses for RNA-sequencing and microarray studies. Nucleic Acids Res 43, e47.

Rodier, F., Coppe, J.P., Patil, C.K., Hoeijmakers, W.A., Munoz, D.P., Raza, S.R., Freund, A., Campeau, E., Davalos, A.R., and Campisi, J. (2009). Persistent DNA damage signalling triggers senescence-associated inflammatory cytokine secretion. Nat Cell Biol 11, 973-979.

Ruhland, M.K., Loza, A.J., Capietto, A.H., Luo, X., Knolhoff, B.L., Flanagan, K.C., Belt, B.A., Alspach, E., Leahy, K., Luo, J., et al. (2016). Stromal senescence establishes an immunosuppressive microenvironment that drives tumorigenesis. Nat Commun 7 , 11762.

Saida, Y., Watanabe, S., Tanaka, T., Baba, J., Sato, K., Shoji, S., Igarashi, N., Kondo, R., Okajima, M., Koshio, J., et al. (2015). Critical Roles of Chemoresistant Effector and Regulatory T Cells in Antitumor Immunity after Lymphodepleting Chemotherapy. The Journal of Immunology 195, 726-735. 
Sanoff, H.K., Deal, A.M., Krishnamurthy, J., Torrice, C., Dillon, P., Sorrentino, J., Ibrahim, J.G., Jolly, T.A., Williams, G., Carey, L.A., et al. (2014). Effect of cytotoxic chemotherapy on markers of molecular age in patients with breast cancer. J Natl Cancer Inst 106, dju057.

Schafer, M.J., White, T.A., lijima, K., Haak, A.J., Ligresti, G., Atkinson, E.J., Oberg, A.L., Birch, J., Salmonowicz, H., Zhu, Y., et al. (2017). Cellular senescence mediates fibrotic pulmonary disease. Nature Communications 8, 14532.

Scuric, Z., Carroll, J.E., Bower, J.E., Ramos-Perlberg, S., Petersen, L., Esquivel, S., Hogan, M., Chapman, A.M., Irwin, M.R., Breen, E.C., et al. (2017). Biomarkers of aging associated with past treatments in breast cancer survivors. NPJ Breast Cancer 3, 50.

Serrano, M., Lin, A.W., McCurrach, M.E., Beach, D., and Lowe, S.W. (1997). Oncogenic ras Provokes Premature Cell Senescence Associated with Accumulation of p53 and p16INK4a. Cell 88, 593-602.

Sharma, A.K., Roberts, R.L., Benson, R.D., Jr., Pierce, J.L., Yu, K., Hamrick, M.W., and McGee-Lawrence, M.E. (2020). The Senolytic Drug Navitoclax (ABT-263) Causes Trabecular Bone Loss and Impaired Osteoprogenitor Function in Aged Mice. Frontiers in cell and developmental biology 8, 354.

Waggott, D., Chu, K., Yin, S., Wouters, B.G., Liu, F.F., and Boutros, P.C. (2012). NanoStringNorm: an extensible R package for the pre-processing of NanoString mRNA and miRNA data. Bioinformatics (Oxford, England) 28, 1546-1548.

Walraven, M., and Hinz, B. (2018). Therapeutic approaches to control tissue repair and fibrosis: Extracellular matrix as a game changer. Matrix Biology 71-72, 205-224.

Willis, S., Day, C.L., Hinds, M.G., and Huang, D.C.S. (2003). The Bcl-2-regulated apoptotic pathway. Journal of Cell Science 116, 4053-4056.

Xu, M., Pirtskhalava, T., Farr, J.N., Weigand, B.M., Palmer, A.K., Weivoda, M.M., Inman, C.L., Ogrodnik, M.B., Hachfeld, C.M., Fraser, D.G., et al. (2018). Senolytics improve physical function and increase lifespan in old age. Nature Medicine 24, 1246-1256. 
Ye, Q.-F., Zhang, Y.-C., Peng, X.-Q., Long, Z., Ming, Y.-Z., and He, L.-Y. (2012). Silencing Notch-1 induces apoptosis and increases the chemosensitivity of prostate cancer cells to docetaxel through Bcl-2 and Bax. Oncology letters 3, 879-884.

Yosef, R., Pilpel, N., Tokarsky-Amiel, R., Biran, A., Ovadya, Y., Cohen, S., Vadai, E., Dassa, L., Shahar, E., Condiotti, R., et al. (2016). Directed elimination of senescent cells by inhibition of BCL-W and BCL-XL. Nature Communications 7, 11190. 
bioRxiv preprint doi: https://doi.org/10.1101/2020.10.17.343590; this version posted November 9, 2020. The copyright holder for this

preprint (which was not certified by peer review) is the author/funder. All rights reserved. No reuse allowed without permissior Figure 1

a
1
$6-10$
(14)
21
32
37
(119)
Panel b Panel d
Panel c
Q Day $\bigcirc$ chemotherapy or vehicle dose $\bigcirc$ IV tumour cell injection $\bigcirc$ Sacrifice mice

b
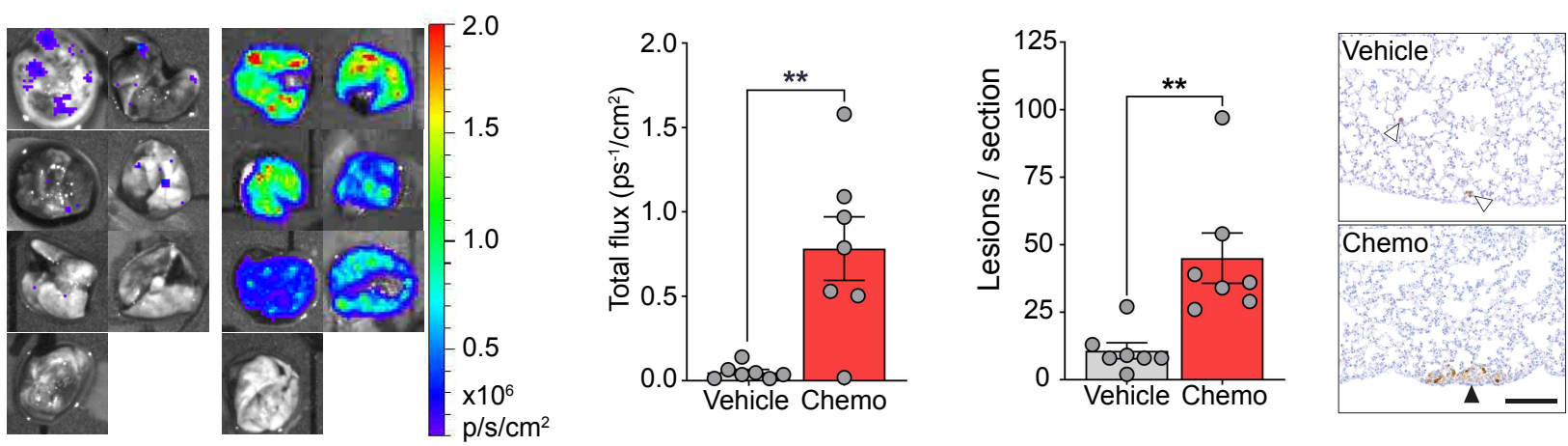

C
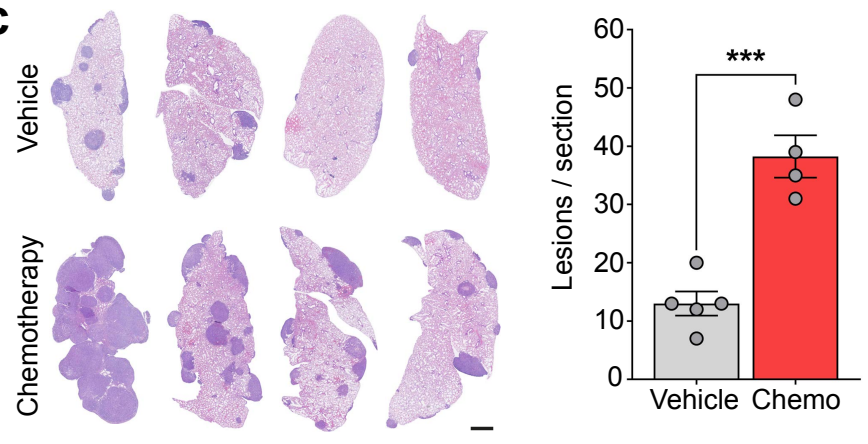

d
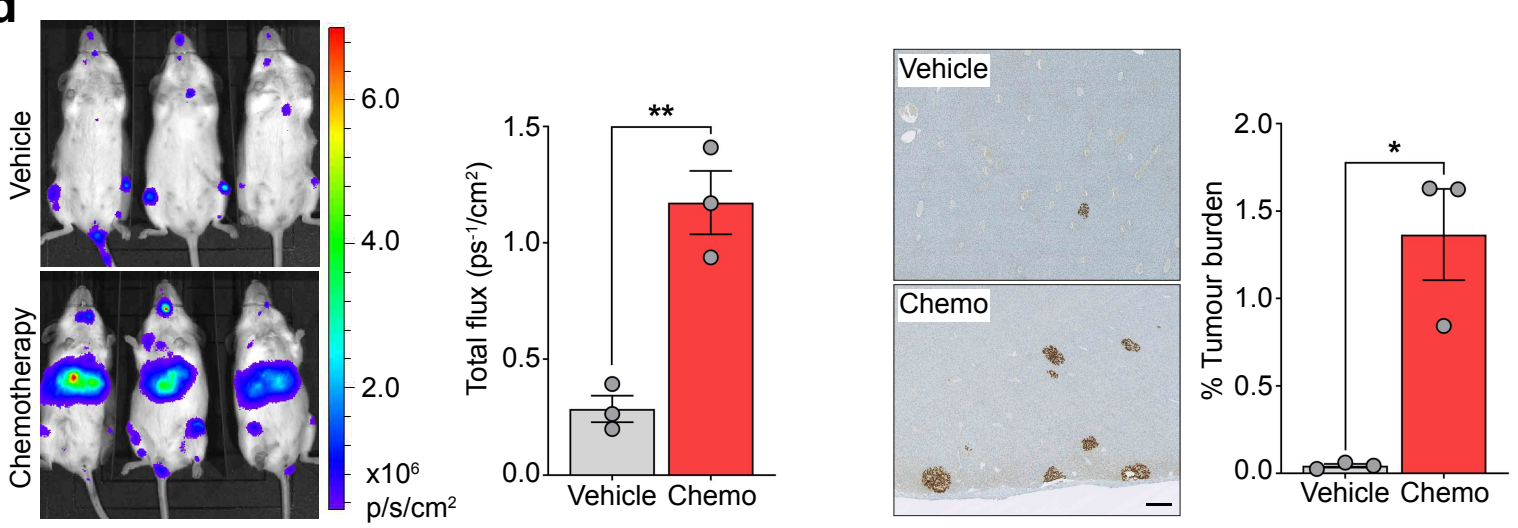
Figure 1 | Prior chemotherapy treatment increases metastatic colonisation. a Schedule of chemotherapy (doxorubicin $2.7 \mathrm{mg} \mathrm{kg}^{-1}$ and cyclophosphamide $43 \mathrm{mg} \mathrm{kg}^{-1}$ ) or vehicle treatment and subsequent intravenous (IV) tumour cell inoculation. b BALB/c mice ( $n=7$ per group) were treated with 4 doses of chemotherapy or vehicle as illustrated in panel a. On Day 21 mice were inoculated via the tail vein with $1 \times 10^{6}$ D2.OR-Luc tumour cells. Mice were sacrificed 11 days after inoculation (Day 32). Shown are ex vivo IVIS images of the lungs, quantification of IVIS signal in the lungs (mean \pm s.e.m, Welch's $t$-test), quantification of the mean number of metastatic lesions from 3 lung sections per mouse (mean \pm s.e.m, Welch's $t$-test), and representative immunohistochemical images of luciferase staining (scale bar, $125 \mu \mathrm{m})$. White arrowheads indicate single disseminated tumour cells, black arrowheads indicate macrometastatic deposits. c BALB/c mice ( $n=4$ or 5 per group) were treated with a schedule of chemotherapy or vehicle as outlined in panel a. On Day 21 mice were inoculated via the tail vein with $1 \times 10^{6}$ D2.OR-Luc tumour cells. Mice were sacrificed 98 days after inoculation (Day 119). Shown are representative H\&E stained sections of the lungs (scale bar, $1 \mathrm{~mm}$ ) and quantification of the mean number of metastatic lesions from 3 lung sections per mouse (mean \pm s.e.m, Student's $t$-test). d NOD Rag gamma (NRG) mice ( $n=3$ per group) were treated with a schedule of chemotherapy or vehicle as illustrated in panel a. Mice were implanted subcutaneously with a $0.36 \mathrm{mg}$ oestrogen pellet on Day 17 . On Day 21, 2 × $10^{6}$ ZR-75-1-Luc human breast tumour cells were inoculated via the tail vein. 15 days after tumour cell inoculation (Day 36), mice were IVIS imaged in vivo (mean \pm s.e.m, Student's t-test), sacrificed 24 hours later (Day 37), and percentage metastatic burden in quantified in 3 liver sections per mouse, based on lamin A/C staining (mean \pm s.e.m, Welch's t-test; scale bar, $250 \mu \mathrm{m})$. 
a

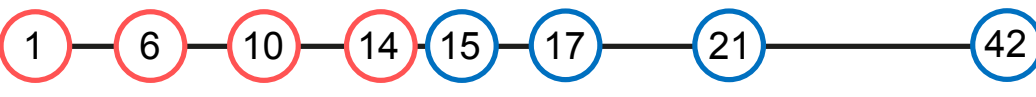

(42)....... (105)

$\otimes$ Day $\bigcirc$ Chemotherapy or vehicle dose $\bigcirc$ sacrifice mice

b
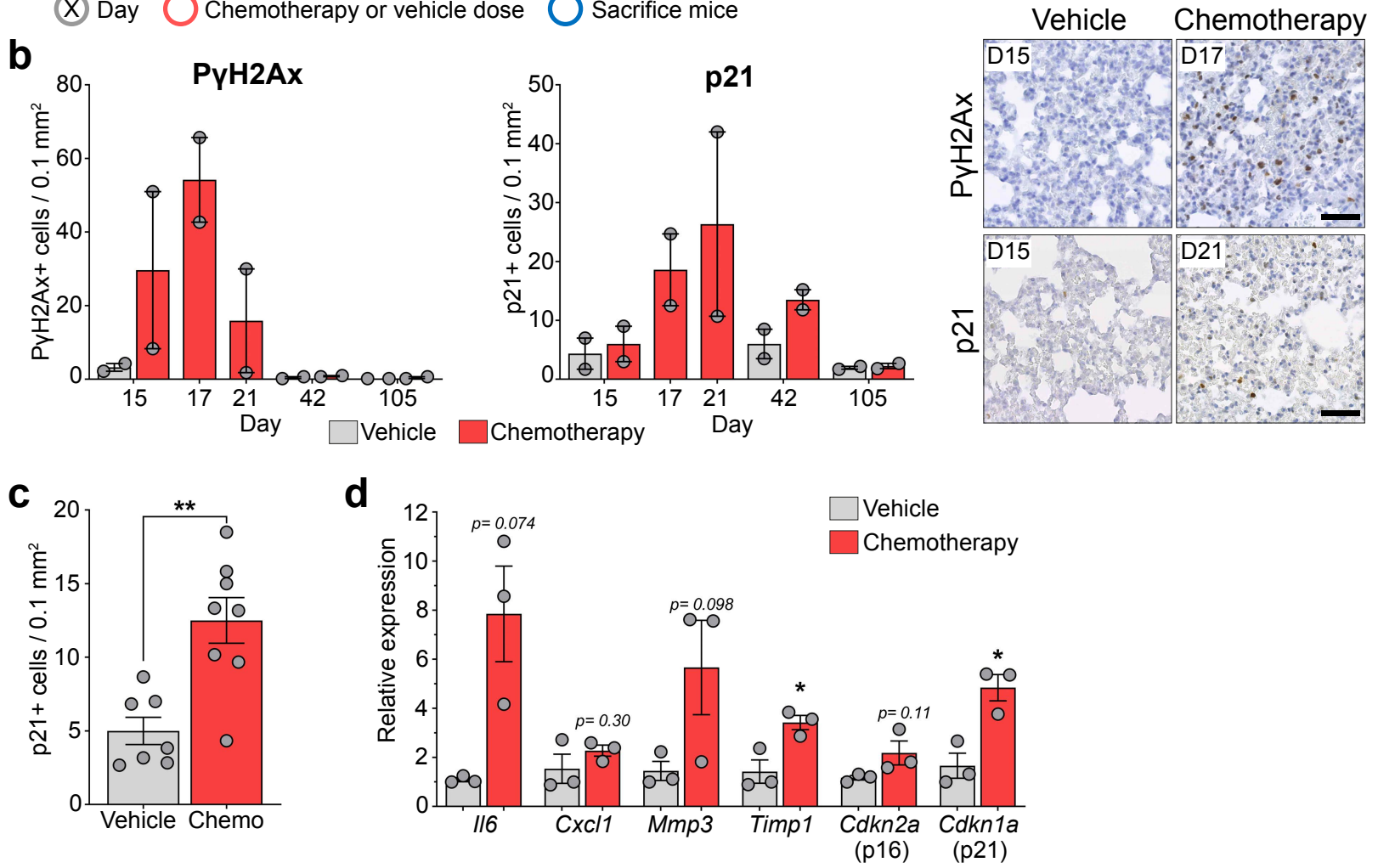

e Vehicle vs. Chemotherapy
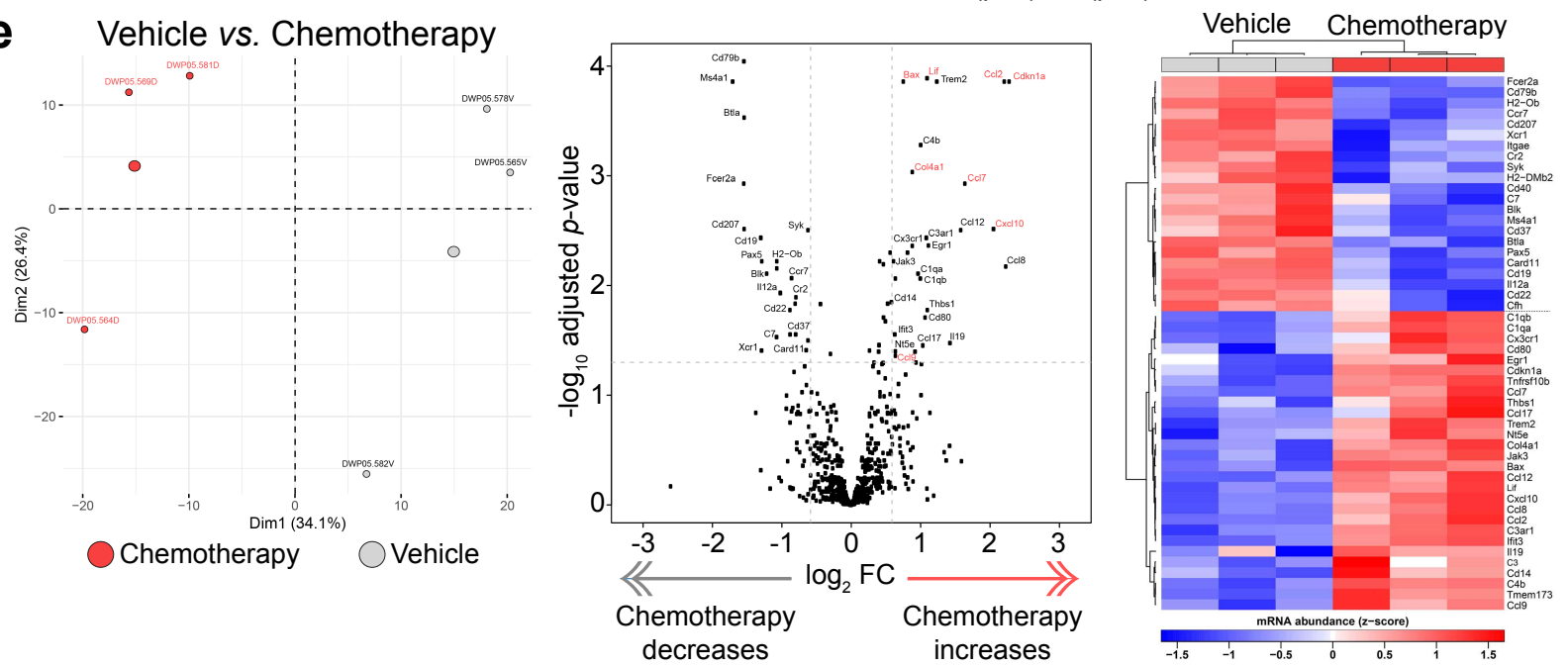
Figure 2 | Systemic chemotherapy treatment induces DNA damage and senescence in normal tissue. a Schedule of the chemotherapy or vehicle treatment and subsequent tissue collection timepoints. b Chemotherapy-treated mice $(n=2)$ were sacrificed at the indicated time points. Vehicle-treated mice $(n=2)$ were sacrificed on Day 15, 42 and 105. Lung sections were stained for phospho-yH2Ax $(\mathrm{PyH} 2 \mathrm{Ax})$ or $\mathrm{p} 21$. Staining was quantified in 6 randomised $0.1 \mathrm{~mm}^{2}$ fields of view per section (mean \pm s.e.m). Shown are representative images of Day $17 \mathrm{PyH} 2 \mathrm{Ax}$ and Day $21 \mathrm{p} 21$ staining from chemotherapy-treated mice, compared to vehicle-treated mice on Day 15 (scale bars, $50 \mu \mathrm{m})$. c BALB/c mice ( $\mathrm{n}=7$ or 8 per group) were treated with 4 doses of chemotherapy or vehicle as illustrated in panel a and mice sacrificed on Day 35. p21 staining in lung tissue was quantified as described in panel b, (mean \pm s.e.m, Student's $t$-test). d,e BALB/c mice ( $n=3$ per group) were treated with 4 doses chemotherapy or vehicle as illustrated in panel a. On Day 21 mice were sacrificed and the lungs snap-frozen. Panel d, RNA was extracted and expression of the indicated genes was measured by RTqPCR (mean \pm s.e.m, Student's $t$-test except $/ / 6$ Welch's $t$-test). Panel e, RNA was freshly isolated from the frozen lung tissue and gene expression analysed using the NanoString mouse PanCancer Immune panel. Left panel, principal component analysis of the 6 samples. Middle panel, volcano plot showing differentially expressed genes between chemotherapy and vehicle treated mice. Genes with absolute $\log _{2}$ fold change $\geq 0.585$ and FDR adjusted $p$-value $<0.05$ were considered significant and shown in the heat map (right panel). 


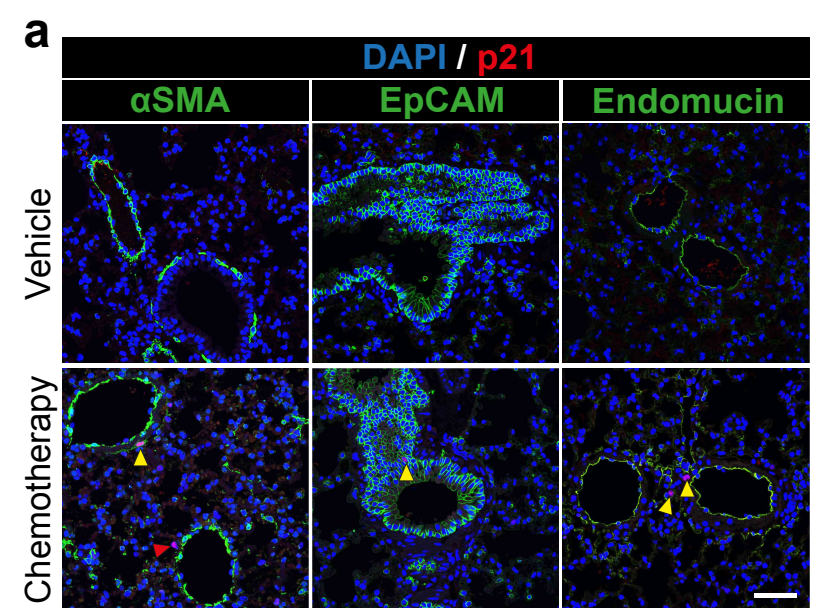

b

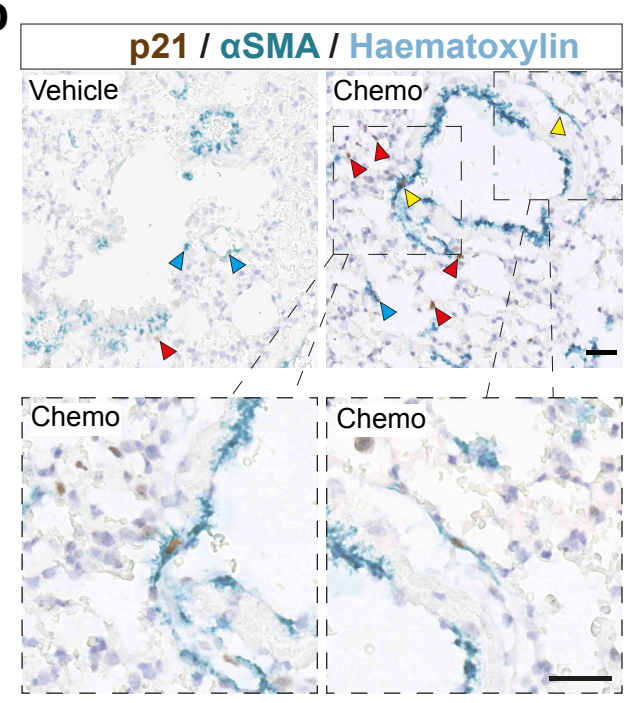

C

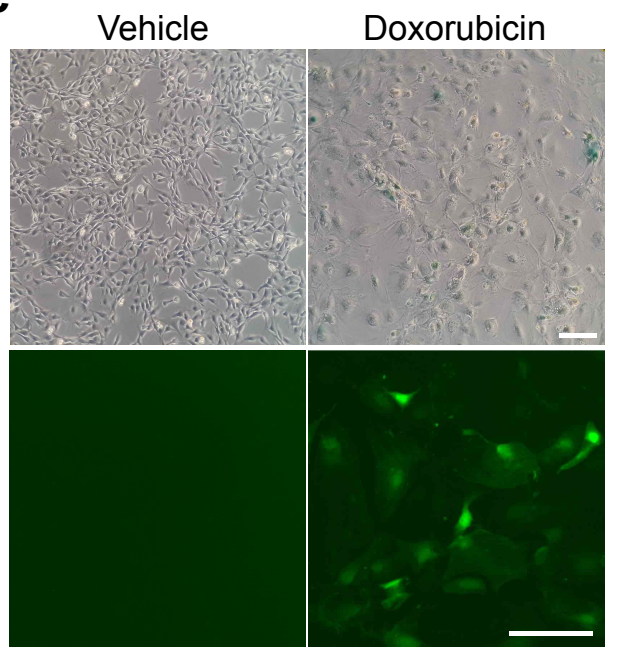

$\square$ Vehicle

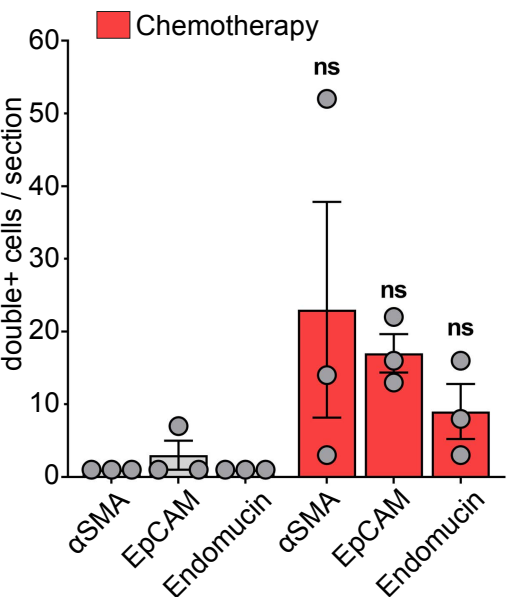

d

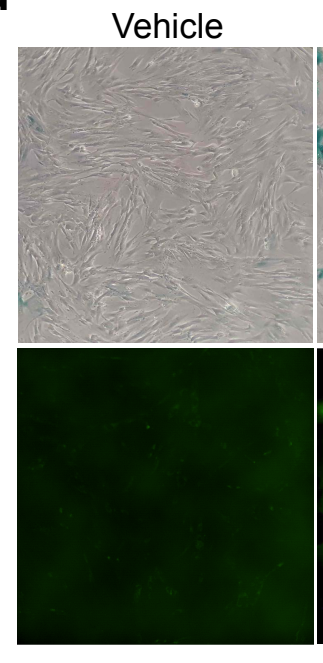

Doxorubicin

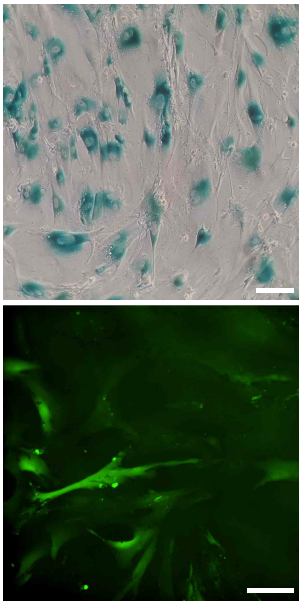

e

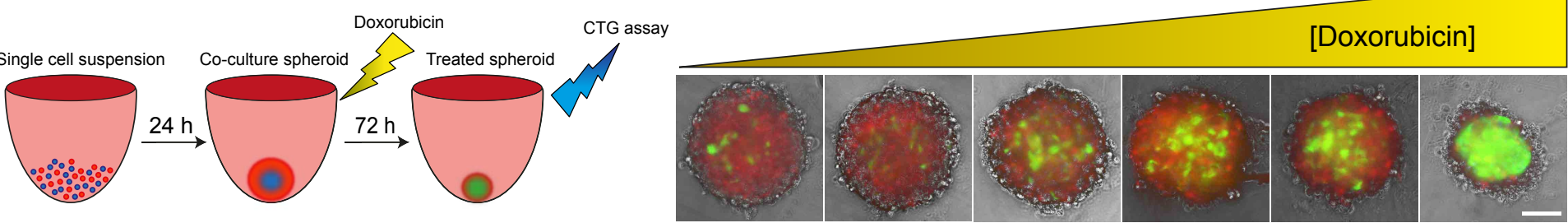


Figure 3 | Chemotherapy-induced fibroblast senescence in vitro and in vivo. a BALB/C mice ( $n=3$ per group) were treated with 3 doses of chemotherapy or vehicle on Day 1, 5 and 9 and sacrificed on Day 16. FFPE lung sections were stained for p21 (red) and DAPI (blue), and either $\alpha$ smooth muscle actin (aSMA), EpCAM or endomucin (green), scale bar, $50 \mu \mathrm{m}$. Red arrowheads indicate p21 single-positive cells, yellow arrowheads indicate doublepositive cells. Staining was quantified by counting all p21+ cells per section (middle panel, mean \pm s.e.m, Welch's $t$-test), then counting double-positive cells (right panel, mean \pm s.e.m, one way ANOVA). b Lung FFPE sections from BALB/c mice (from Figure $2 b$ ) sacrificed 7 days following the final chemotherapy treatment or 1 day following the final vehicle treatment were stained for p21 (brown) and aSMA (cyan) and counterstained with haematoxylin. Blue arrowheads indicate aSMA single-positive cells, red arrowheads indicate p21 single-positive cells, yellow arrowheads indicate double positive cells. Left panels; scale bar, $50 \mu \mathrm{m}$. Lower panels; higher magnification views of indicated regions; scale bar, $25 \mu \mathrm{m}$. c Mouse 3T3-146a fibroblasts were treated with $170 \mathrm{nM}$ doxorubicin for 24 hours, then drugs were removed. 20 days later cells were fixed and stained using the SA- $\beta$ Gal staining kit or expression of the GFP senescence reporter was visualised under a fluorescence microscope (scale bars, $200 \mu \mathrm{m}$ ). Vehicle-treated fibroblasts were seeded 3 days prior to staining or imaging alongside chemotherapy-treated fibroblasts. d Human lung MRC5-146a fibroblasts were treated with $1.7 \mu \mathrm{M}$ doxorubicin for 24 hours, then drugs were removed. 21 days later cells were fixed and stained using the SA- $\beta$ Gal staining kit or GFP expression was visualised under a fluorescence microscope (scale bars, $200 \mu \mathrm{m}$ ). Vehicle-treated fibroblasts were seeded 3 days prior to staining or imaging alongside chemotherapy-treated fibroblasts. e 1,000 D2A1-RFP mouse tumour cells were co-seeded with 1,000 MRC5-146a human fibroblasts into ultra-low attachment U-bottom 96-well plates to form co-culture spheroids. After 24 hours spheroids were treated with doxorubicin (0 - $850 \mathrm{nM}) .72$ hours later, spheroids were imaged (scale bar, $100 \mu \mathrm{m}$.) 
a

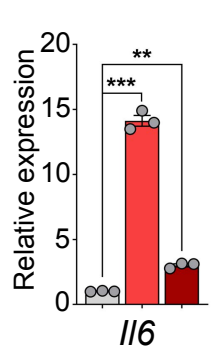

$\square$ Vehicle
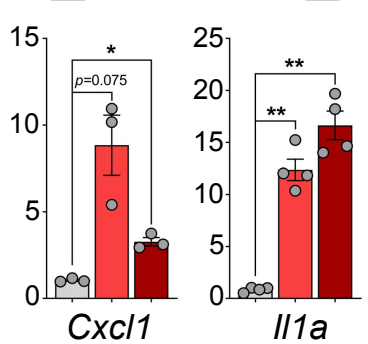

II1a
Doxorubicin 7 days

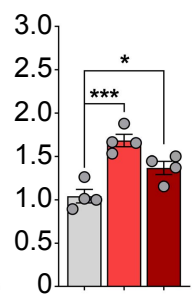

lgf1

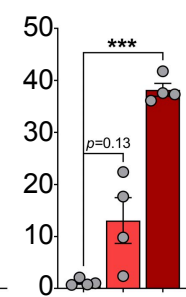

Cdkn1a
Doxorubicin 20 days

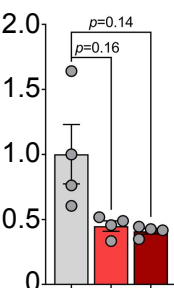

Cdkn1b

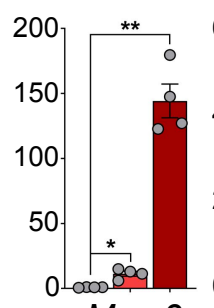

Mmp3
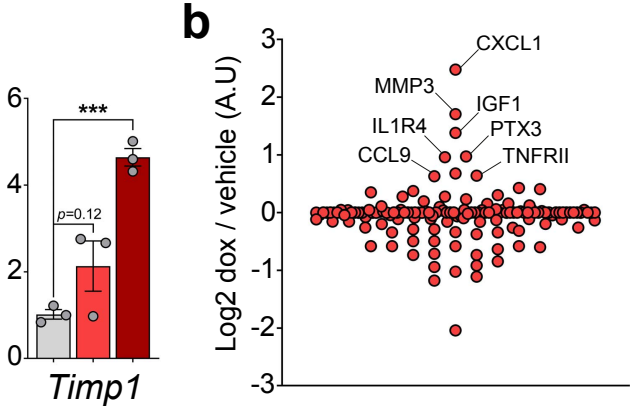

C
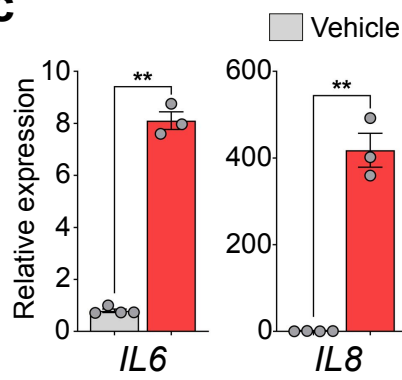

Doxorubicin

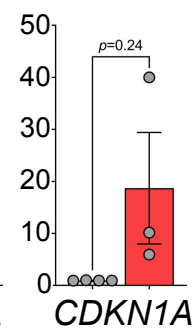

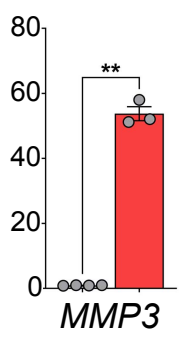

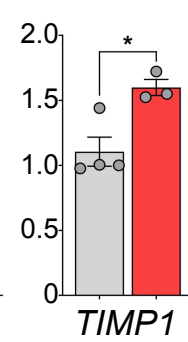

d

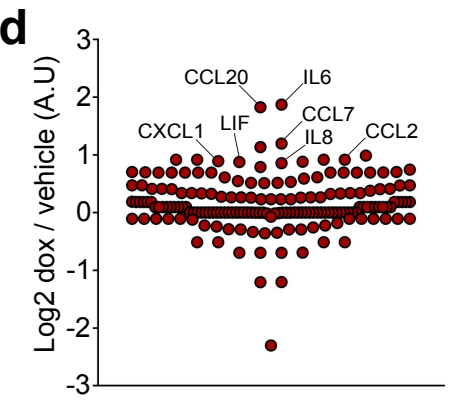

e

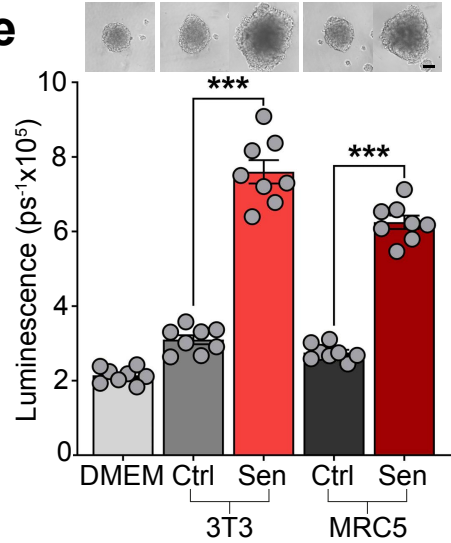

f

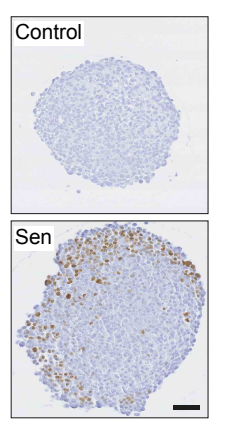

g

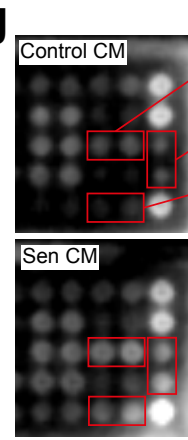

Control 3T3 CM

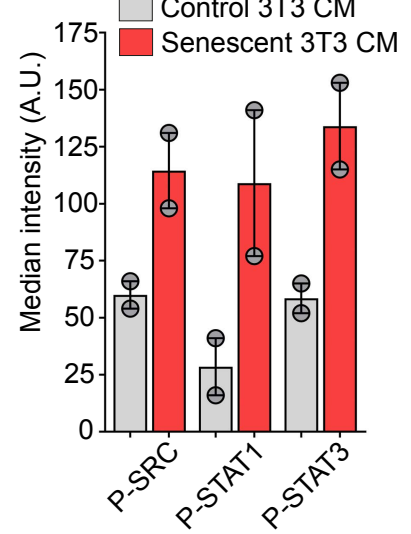


Figure 4 | In vitro characterisation of therapy-induced fibroblast senescence. a 3T3146a fibroblasts were treated with $170 \mathrm{nM}$ doxorubicin for 24 hours. 7 or 20 days after drug withdrawal RNA was extracted from vehicle or doxorubicin-treated fibroblasts for RTqPCR analysis (mean \pm s.e.m, one-way ANOVA for individual genes). b 3T3-146a fibroblasts were treated with doxorubicin as described in panel a. Serum-free CM collected 20 days after doxorubicin withdrawal or from vehicle-treated fibroblasts was analysed using G2000 cytokine arrays. c Human lung MRC5-146a fibroblasts were treated with $1.7 \mu \mathrm{M}$ doxorubicin for 24 hours. 13 days after doxorubicin withdrawal RNA was extracted from vehicle or doxorubicin-treated fibroblasts for RTqPCR analysis (mean \pm s.e.m, Student's $t$-test for individual genes). d Serum-free CM collected from doxorubicin-treated MRC5 fibroblasts 20 days after doxorubicin withdrawal or from vehicle-treated fibroblasts was analysed using G2000 cytokine arrays. e 2,000 D2A1 tumour cells per well were seeded into ultra-low attachment U-bottom 96-well plates in DMEM plus 2\% FBS. After 24 hours, spheroids were incubated with DMEM supplemented with $2 \%$ FBS or CM from control or doxorubicin-senescent 3T3-146a mouse fibroblasts or MRC5-146a human fibroblasts supplemented with $2 \%$ FBS. After 6 days, spheroid viability was measured using the CellTiter-Glo assay ( $n=8$ spheroids per condition, mean \pm s.e.m, one-way ANOVA). Equivalent results were obtained in $>3$ independent experiments. Representative phase contrast images of spheroids are shown above (scale bar, $100 \mu \mathrm{m})$. f D2A1 spheroids were treated with control or senescent 3T3-146a CM as described in panel e and collected after 4 days incubation. FFPE sections from embedded spheroids were stained for Ki67 (scale bar, $50 \mu \mathrm{m}) . \mathbf{g}$ 12,500 D2A1 cells per well were seeded into U-bottom 96-well plates in DMEM plus $2 \%$ FBS and incubated for 48 hours. Spheroids were serum-starved for 1 hour, and then incubated for 30 minutes at $37^{\circ} \mathrm{C}$ with serum-free control or senescent $3 \mathrm{T3}-146 \mathrm{a}$ fibroblast CM for 30 minutes. Protein was extracted and analysed on phosphoprotein antibody arrays slides (30 $\mu \mathrm{g}$ total protein per sample). Median intensity of each target antibody spot was quantified. Shown are cropped images of the relevant portion of the array. Bar graphs show mean of the two replicate target antibody spots (mean \pm s.e.m). 
bioRxiv preprint doi: https://doi.org/10.1101/2020.10.17.343590; this version posted November 9, 2020. The copyright holder for this preprint (which was not certified by peer review) is the author/funder. All rights reserved. No reuse allowed without permissjen;

a

\begin{tabular}{|c|c|c|c|c|}
\hline Senolytic agent & Drug name & \multicolumn{3}{|c|}{ Drug Targets } \\
\hline Navitoclax & ABT263 & BCL2 & BCLxl & BCLw \\
\hline Venetoclax & ABT199 & & BCL2 & \\
\hline UMI-77 & UMI-77 & & MCL1 & \\
\hline A155 & A-1155463 & & BCLxI & \\
\hline
\end{tabular}

C

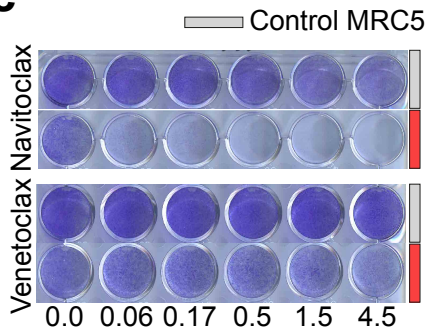

[Senolytic] $(\mu \mathrm{M})$ b

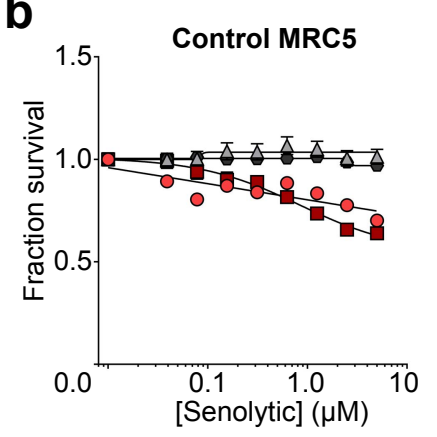

d

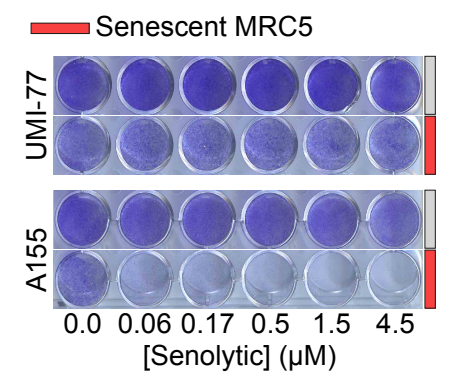

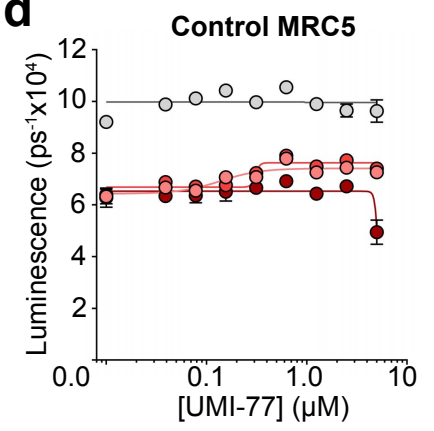

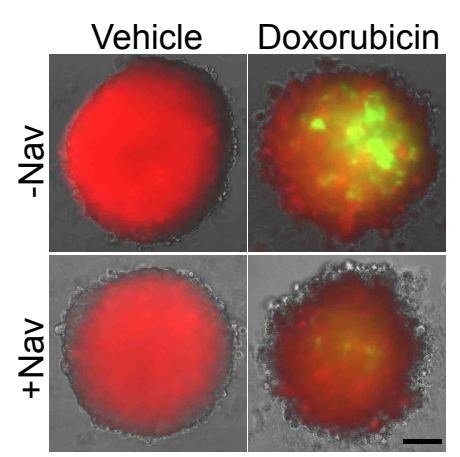

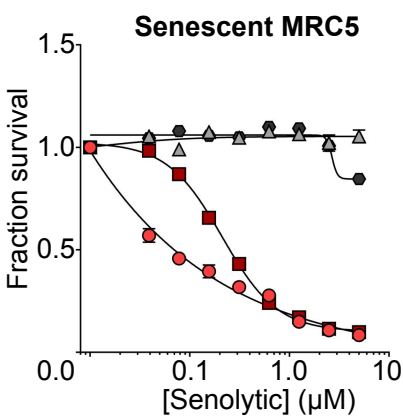

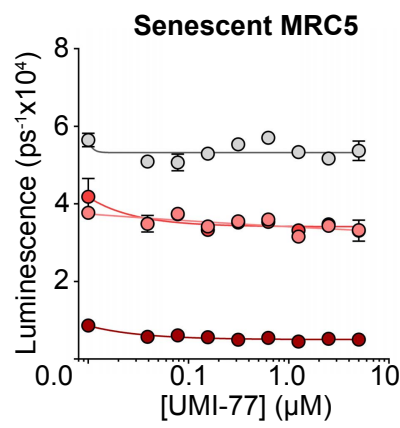

Vehicle $0.1 \mu \mathrm{M} N a v$ $0.3 \mu \mathrm{M}$ Nav $1.0 \mu \mathrm{M} \mathrm{Nav}$

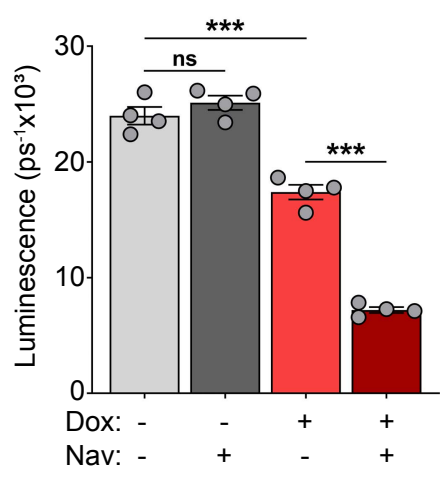




\section{Figure 5 | Senolytic drugs eliminate chemotherapy-induced senescent fibroblasts in}

vitro. a Table detailing senolytic drugs used in the study. b MRC5-146a fibroblasts were treated with $1.7 \mu \mathrm{M}$ doxorubicin for 24 hours. 9 days after treatment withdrawal, control or doxorubicin-senescent fibroblasts were plated into 96-well plates (see methods; $n=3$ wells per condition). After 24 hours fibroblasts were treated with senolytic agents as indicated, and incubated for 72 hours before cell viability was measured by CellTiter-Glo. c Doxorubicinsenescent MRC5 fibroblasts, 10 days following treatment withdrawal, or control fibroblasts were seeded into 24 -well plates (see methods; $n=1$ well per condition), then 24 hours later treated senolytic agents as indicated. After 72 hours, plates were fixed and stained with crystal violet. d Doxorubicin-senescent MRC5 fibroblasts, 9 days following treatment withdrawal, or control fibroblasts were plated into 96-well plates ( $n=3$ wells per condition). After 24 hours fibroblasts were treated with a range of concentrations of UMI-77, alone or in combination with 3 concentrations of navitoclax, and incubated for 72 hours before cell viability was measured by CellTiter-Glo assay. e 5,000 D2A1-RFP mouse tumour cells were co-seeded into ultra-low attachment U-bottom 96-well plates with 5,000 MRC5-146a human fibroblasts ( $n=4$ wells per condition). After 24 hours spheroids were treated with $170 \mathrm{nM}$ doxorubicin or vehicle for 72 hours, followed by treatment with $4.5 \mu \mathrm{M}$ navitoclax or vehicle for a further 72 hours. At the end of the assays spheroids were imaged (scale bar, $100 \mu \mathrm{m}$ ) and cell viability was quantified by CellTiter-Glo (one-way ANOVA). b-e Equivalent results were obtained in 2 - 4 independent experiments. 
bioRxiv preprint doi: https://doi.org/10.1101/2020.10.17.343590; this version posted November 9, 2020. The copyright holdefor this

preprint (which was not certified by peer review) is the author/funder. All rights reserved. No reuse allowed without permispolgure 6

a
(1)- $6-10$
(13) 14
(15) (17)
(20)
$\otimes$ Day $\begin{array}{r}\text { Chemotherapy } \\ \text { or vehicle dose }\end{array}$
Navitoclax
or vehicle dose
IV tumour cell injection $\bigcirc$ sacrifice mice

b

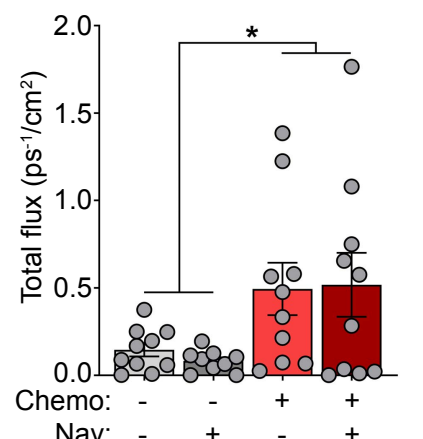

C Vehicle Chemo

-Nav

$+\mathrm{Nav}$

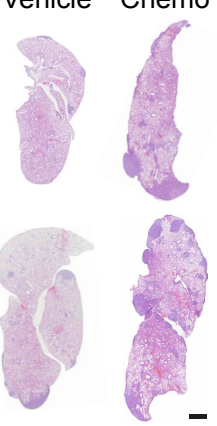

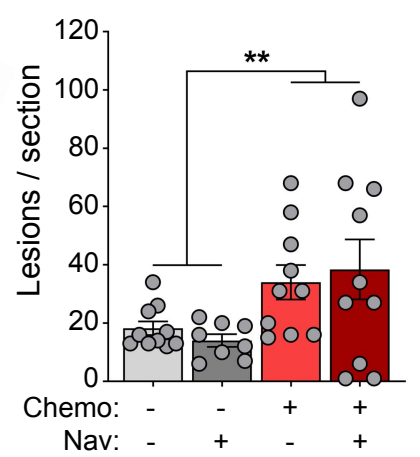

(20)

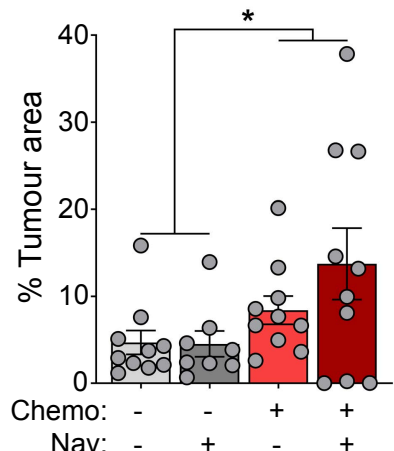

d

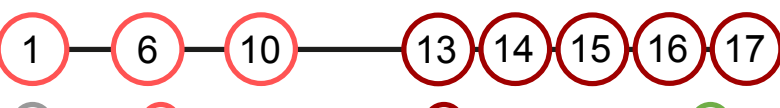

X Day Chemotherapy or vehicle dose

or vehicle dose

e
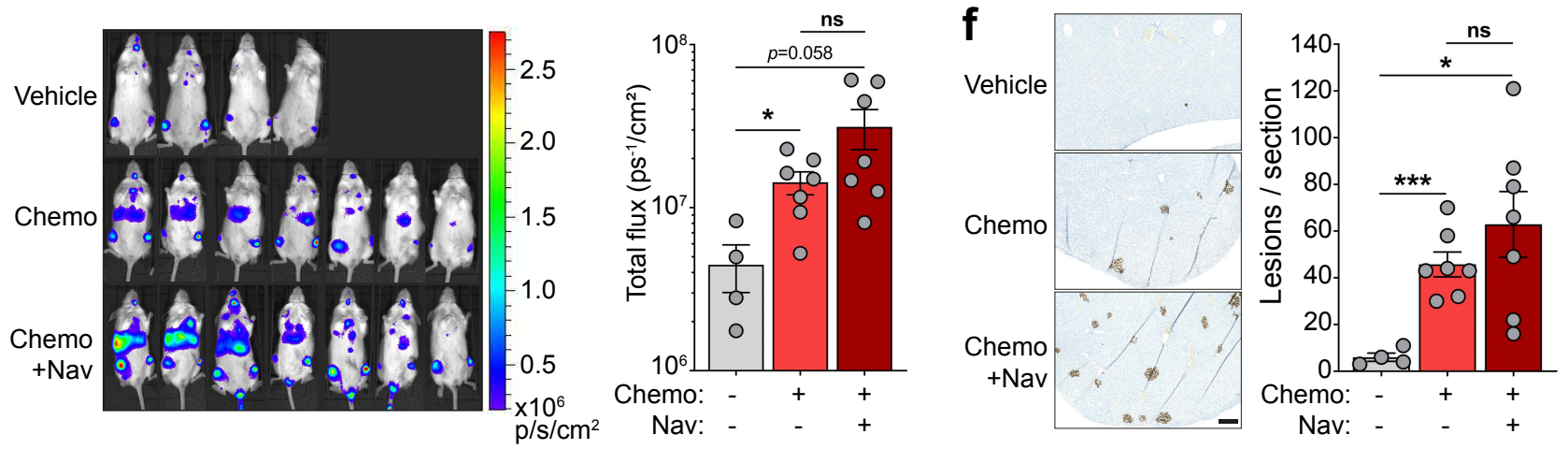

g
(1) 8
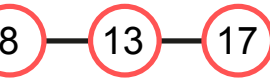
(20) 21 (22)
(23) (24)
Survival analysis
X Day IV tumour cell injection
Chemotherapy
Navitoclax

h
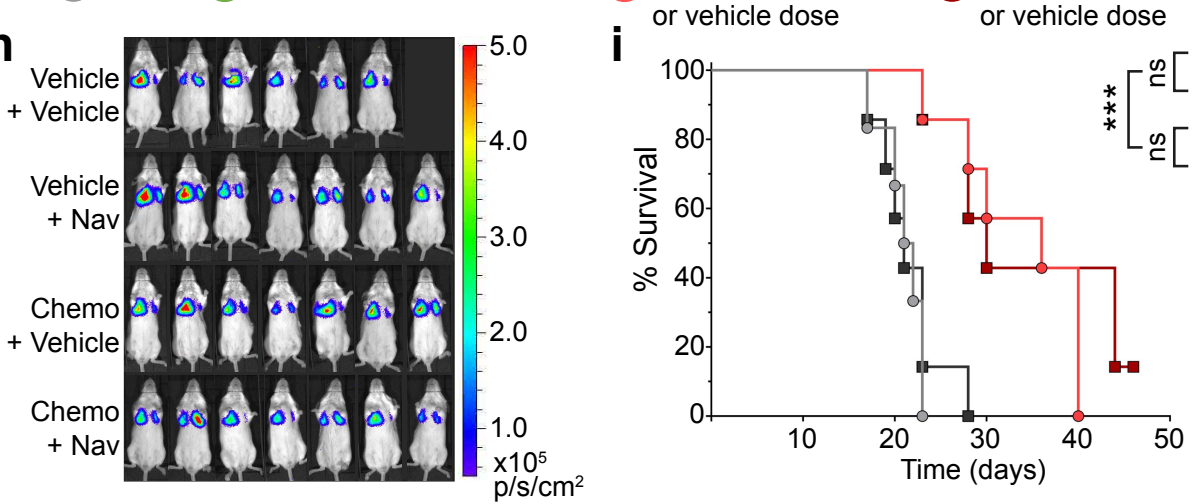

j

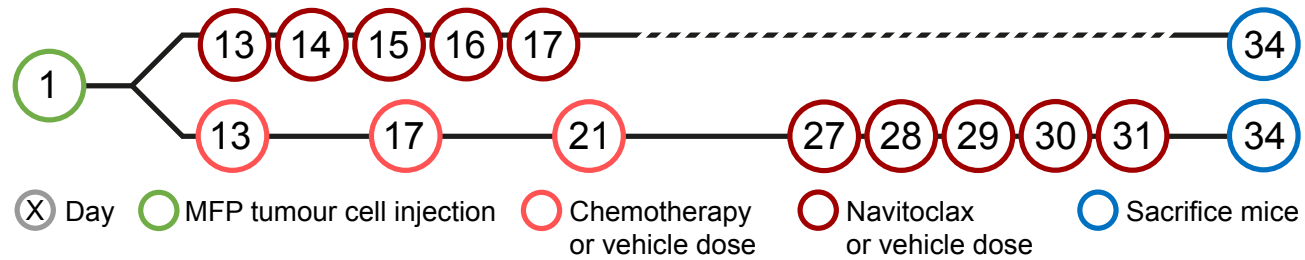

k

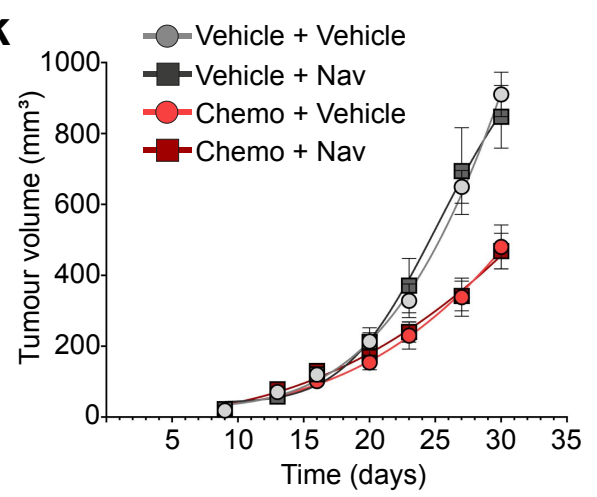

or vehicle dose

or vehicle dose

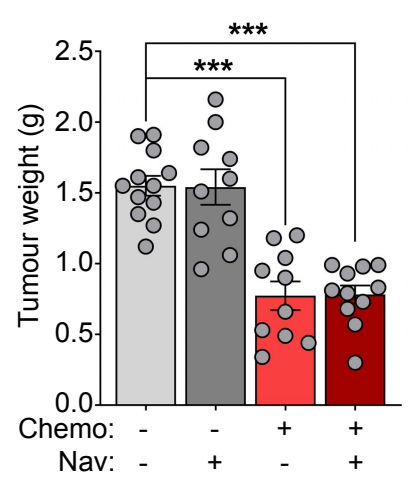

I Vehicle Chemo

$-\mathrm{Nav}$

$+\mathrm{Nav}$

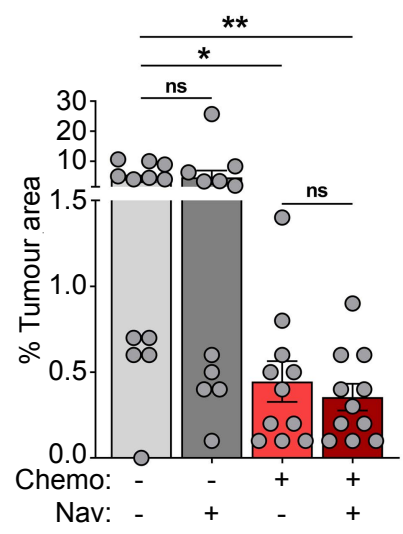




\section{Figure 6 | Effect of combined chemotherapy and navitoclax treatment on metastasis}

and survival. a Schedule for treatment of BALB/c mice with chemotherapy (Chemo) or vehicle followed by navitoclax (Nav) or vehicle, and subsequent intravenous (IV) inoculation of $1 \times 10^{6}$ D2.OR-mChLuc2 tumour cells ( $n=9-10$ mice per group). $\mathbf{b}$ Quantification of in vivo IVIS imaging of the thoracic region on Day 77 (mean \pm s.e.m, Mann-Whitney U-test between vehicle and chemotherapy groups combined). c Quantification of lung metastatic burden at termination of the experiment (Day 91). Left panel, representative H\&E lung sections (scale bar, $1 \mathrm{~mm}$ ). Middle panel, number of metastatic lesions per lung section. Right panel, percentage tumour burden in the lungs (mean \pm s.e.m, Mann-Whitney U-tests between vehicle and chemotherapy groups combined). d Schedule for treatment of NOD Rag gamma mice and subsequent IV inoculation of $2 \times 10^{6}$ ZR-75-1-mChLuc2 tumour cells. e In vivo IVIS quantification of mice ( $n=4$ mice vehicle group, $n=7$ mice Chemo and Chemo + Nav groups) on Day 34 ( \pm s.e.m, Welch's ANOVA). f Representative lamin A/C staining in the liver (scale bar, $250 \mu \mathrm{m}$ ) and quantification of metastatic lesions in the liver (mean \pm s.e.m, Welch's ANOVA). $\mathbf{g}$ Experimental timeline of BALB/c mice inoculated intravenously on Day 1 with $5 \times 10^{5}$ D2A1-mChLuc2 tumour cells ( $n=6-7$ mice per group) followed by chemotherapy (Chemo), navitoclax (Nav) or vehicle treatments. h IVIS images were taken $\sim 90$ minutes after tumour cell injection. i Kaplan-Meier survival analysis. j Experimental timeline of BALB/c mice inoculated orthotopically (4th mammary fat pad) on Day 1 with $2 \times 10^{5}$ D2A1$\mathrm{m} 2$ tumour cells. $\mathbf{k}$ Primary tumour growth was measured twice weekly until the termination of the experiment (left panel) and tumour weight measured at necropsy on Day 34 ( \pm s.e.m, one-way ANOVA). I Quantification of spontaneous metastasis to the lungs in 3 lung sections per mouse (mean \% tumour burden per lung section \pm s.e.m, non-parametric one-way ANOVA). Representative images of lung H\&E stained sections are shown, arrowheads indicate metastatic deposits (scale bar, $1 \mathrm{~mm}$ ). 
a
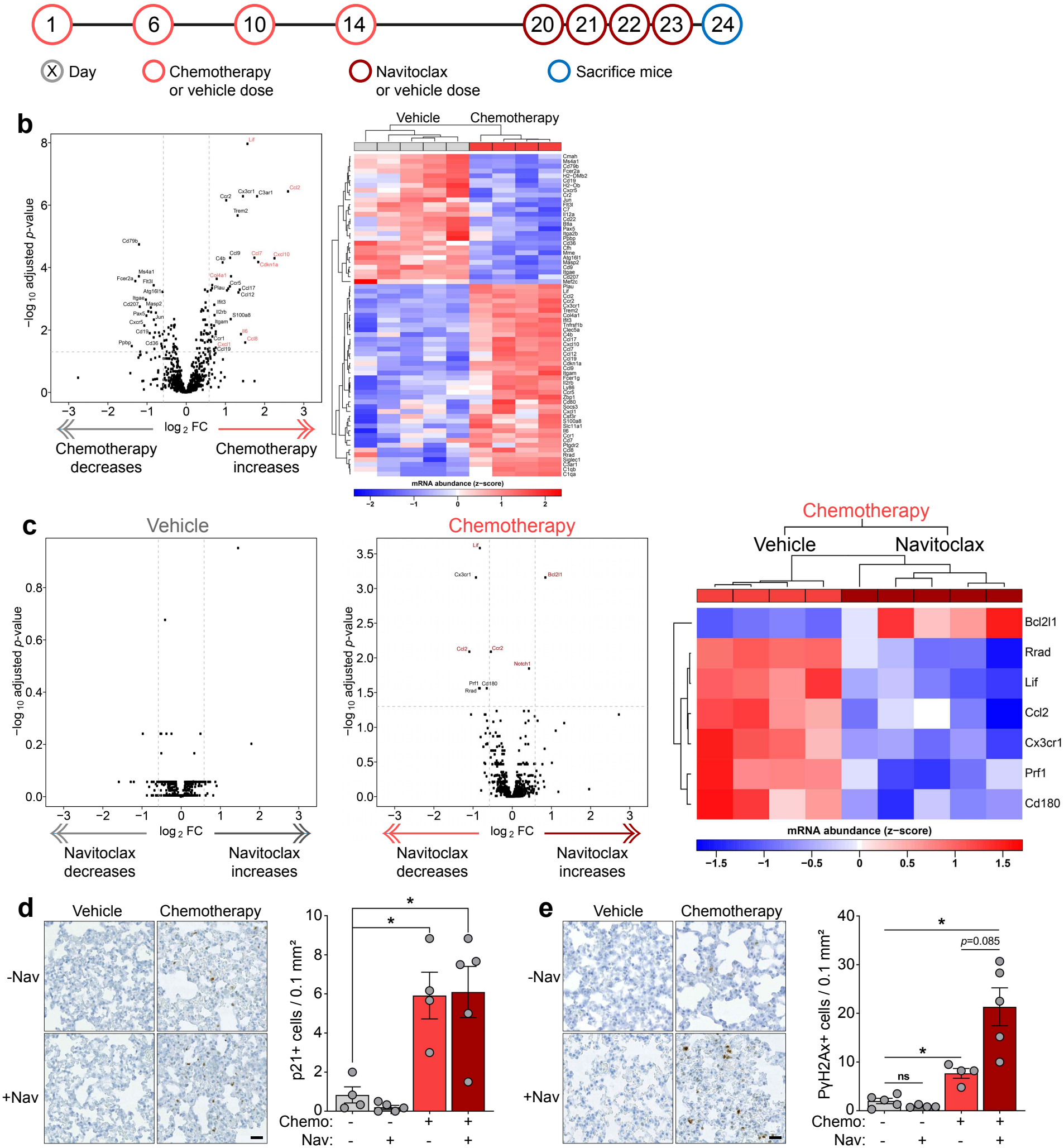


\section{Figure 7 | Chemotherapy-induced senescent cells are not eliminated by navitoclax in}

vivo. a Schedule for the treatment of BALB/c mice with chemotherapy (Chemo) or vehicle followed by navitoclax (Nav) or vehicle. The experiment was terminated on Day 24. b,c RNA was extracted from frozen lung tissue and gene expression profiling performed using the NanoString mouse PanCancer Immune panel. Normalisation was carried out as described in Figure 2e. Panel b, volcano plot showing differentially expressed genes between chemotherapy and vehicle treated mice (left panel). Genes with absolute $\log _{2}$ fold change $\geq$ 0.585 and FDR adjusted $p$-value $<0.05$ were considered significant and shown in the heat map (right panel). Panel c, volcano plot showing differentially expressed genes between vehicle-treated mice \pm navitoclax (left panel), or chemotherapy-treated mice \pm navitoclax (middle panel). Genes with absolute $\log _{2}$ fold change $\geq 0.585$ and FDR adjusted $p$-value $<$ 0.05 were considered significant and shown in the heat map (right panel). No heat map is shown for vehicle \pm navitoclax samples since no hits reached the threshold for significance. d,e In parallel, lung tissue was stained for $\mathrm{PyH} 2 \mathrm{Ax}$ and p21 (scale bars, $25 \mu \mathrm{m}$ ) and quantified as described in Figure $2 b$ (Welch's ANOVA). 